\title{
Single-cell analysis of colonic epithelium reveals unexpected shifts in cellular composition and molecular phenotype in treatment-naïve adult Crohn's disease
}

Matt Kanke ${ }^{1,2}$, Meaghan M. Kennedy ${ }^{3-5}$, Sean Connelly ${ }^{3}$, Matthew Schaner ${ }^{3}$, Michael T. Shanahan ${ }^{1}$, Elisabeth A. Wolber ${ }^{3}$, Caroline Beasley ${ }^{3}$, Grace Lian $^{3}$, Animesh Jain ${ }^{3}$, Millie D. Long ${ }^{3}$, Edward L. Barnes ${ }^{3}$, Hans H. Herfarth ${ }^{3}$, Kim L. Isaacs ${ }^{3}$, Jonathan J. Hansen ${ }^{3}$, Muneera Kapadia ${ }^{6}$, José Gaston Guillem ${ }^{6}$, Terrence S. Furey ${ }^{3-5,7,}$, Shehzad Z. Sheikh ${ }^{3-5,{ }^{*}}$, and Praveen Sethupathy ${ }^{1-2,{ }^{*}}$

${ }^{1}$ Department of Biomedical Sciences, ${ }^{2}$ Center for Vertebrate Genomics, Cornell University, Ithaca, NY, USA, ${ }^{3}$ Center for Gastrointestinal Biology and Disease, ${ }^{4}$ Department of Genetics, ${ }^{5}$ Curriculum in Bioinformatics and Computational Biology, ${ }^{6}$ Department of Surgery,

${ }^{7}$ Department of Biology, University of North Carolina at Chapel Hill, Chapel Hill, NC, USA

*Correspondence: P.S. (pr46@cornell.edu), S.Z.S. (shehzad sheikh@med.unc.edu), T.S.F (tsfurey@email.unc.edu)

Abstract: The intestinal epithelial barrier is comprised of a monolayer of specialized intestinal epithelial cells (IECS) that are critical in maintaining gut mucosal homeostasis. Dysfunction within various IEC fractions can increase intestinal permeability, resulting in a chronic and debilitating condition known as Crohn's disease (CD). Defining the molecular changes in each IEC type in CD will contribute to an improved understanding of the pathogenic processes and the identification of potential therapeutic targets. Here we performed, for the first time at single-cell resolution, a direct comparison of the colonic epithelial cellular and molecular landscape between treatment-naïve adult CD and non-IBD control patients. Our analysis revealed that in $C D$ patients there is a significant skew in the colonic epithelial cellular distribution away from canonical LGR5+ stem cells, located at the crypt-bottom, and toward one specific subtype of mature colonocytes, located at the crypt-top. Further analysis revealed unique changes to gene expression programs in every major cell type, including a previously undescribed suppression in CD of most enteroendocrine driver genes as well as L-cell markers including GCG. We also dissect a previously poorly understood SPIB+ cell cluster, revealing at least four sub-clusters that exhibit unique features. One of these $S P I B+$ sub-clusters expresses crypt-top colonocyte markers and is significantly up-regulated in $C D$, whereas another subcluster strongly expresses and stains positive for lysozyme (albeit no other canonical Paneth cell marker), which surprisingly is greatly reduced in expression in CD. Finally, through integration with data from genome-wide association studies, we show that genes implicated in CD risk exhibit heretofore unknown cell-type specific patterns of aberrant expression in $C D$, providing unprecedented insight into the potential biological functions of these genes.

Keywords: Crohn's disease, single-cell, epithelium, colonocyte, gene expression, ISC, EEC, SPIB 


\section{Introduction}

The colonic epithelium acts as an essential barrier between the luminal contents of the colon, including a diverse compendium of microbes, and the underlying lamina propria immune system. The epithelium is a heterogenous mix of distinct cell types with a wide range of specialized functions, including absorptive cells that transport nutrients and electrolytes (colonocytes), and secretory cells that emit factors such as mucins (goblet cells) and endocrine hormones (enteroendocrine cells). Stem cells at the base of the colonic crypt are responsible for the continual, rapid renewal of this epithelial layer. Each cell type is crucial for the maintenance of intestinal homeostasis and defects in any could contribute to the onset of inflammatory bowel disease (IBD).

IBD consists of two main disease types, ulcerative colitis (UC) and Crohn's disease (CD), and is characterized by chronic intestinal inflammation that can lead to severe tissue damage and organ dysfunction. Despite recent advances, the etiology of IBD still remains largely unknown. Unrelenting inflammation is attributed to a complex interaction between genetic, luminal (microbial), and environmental factors that trigger an inappropriate mucosal immune response. Recent studies have begun to unravel the role of different colonic epithelial cell types in IBD using single-cell RNA sequencing (scRNA-seq) (Elmentaite et al., 2020; Parikh et al., 2019; Smillie et al., 2019). While they have advanced our understanding, important challenges remain to be addressed. Notably, the patients included in the studies exhibit varying disease durations, and some have been treated with therapeutics, which are confounding variables. No scRNA-seq study to date has investigated treatment-naïve adult IBD. Moreover, the studies have focused mostly on patients with UC. No scRNA-seq study to date has performed a focused investigation of colonic epithelium in adult CD. Changes to the relative abundance and molecular character of different colonic epithelial cell types during CD pathogenesis is poorly understood and merits deeper examination.

To investigate this, we performed single-cell transcriptional profiling in epithelial cells from a cohort of treatment-naïve adult CD patients $(n=3)$ and healthy controls $(n=4)$. Our analysis reveals that the colonic epithelium of CD patients exhibits significant and unexpected shifts in cellular composition as well as cell-type specific transcriptional profiles, providing important clues about the early molecular events that promote the dysfunction of this critical tissue during $C D$ pathogenesis.

\section{Results}

Single-cell analysis provides a high resolution picture of the colonic epithelium from adult nonIBD and Crohn's patients

We harvested mucosal tissue from the ascending colon of treatment-naïve adult individuals with Crohn's disease ( $C D, n=3$ ) and non-IBD healthy controls (NIBD, n=4) (Table S1). We then enriched for epithelial cells, which were dissociated into single-cell suspensions 
(Methods). Samples were subjected to RNA sequencing using the 10X Chromium platform (Methods) and 13,039 cells remained after filtering (Methods).

We processed and analyzed the data (CD and NIBD together) using Cell Ranger and Seurat (Methods) and visualized the cell clusters using Uniform Manifold Approximation and Project (UMAP)(Becht et al., 2018). We identified 21 cell clusters (Figure S1A), 7 of which were determined to be different types of immune cells (Figure S1B), representing $21 \%$ of the total. We removed these cells and re-clustered the remaining 10,162 epithelial cells, resulting in 14 cell clusters. Based on the expression levels of previously annotated marker genes (Smillie et al., 2019), we assigned these clusters to 14 different colonic epithelial cell types (Figure 1A,B). These cell types include LGR5+ stem cells; $M U C 2+$ immature and mature goblet cells; $C H G A+$ enteroendocrine cells (EECs); three categories of cycling cells including both G2-M-G1 and Sphase transit-amplifying (TA) cells, secretory progenitors (SPs), colonocyte progenitors; and at least four other cell clusters expressing known markers of colonocytes including $C A 1+$ early and late colonocytes, CEACAM7+ colonocytes, and SPIB+ cells (Figure 1B,C). We found that each of these cell types express a unique set of markers (Methods, Figure 1D). Specifically, the SPIB+ cells are uniquely marked by SPIB, NOTCH2 and HES4; the CEACAM7+ cells uniquely express several genes including one long, non-coding RNA (LINCO1133) that has been implicated previously in cancer and in the regulation of the Wnt signaling pathway (Yang et al., 2018); and the $C A 1+$ late colonocytes share MALL expression with CEACAM7+ cells but uniquely express CA1 (Figure 1D).

Colonic epithelial cells from the crypt-bottom to the crypt-top represent a gradient of maturation, with stem/progenitor cells at the bottom and mature differentiated colonocytes at the top. Using a previously defined 15-gene signature (Parikh et al., 2019) we computed a cryptaxis score for every cell in each cluster (Figure 1E), which shows good correspondence between our cluster annotations and known cell-type positions within the crypt. RNA velocity analysis (La Manno et al., 2018) confirmed that the dataset represents the full maturational spectrum of the colonic epithelium (Figure 1F).

The colonic epithelial cellular landscape is skewed toward a crypt-top signature in Crohn's disease

Next we assessed CD and NIBD data separately to determine whether the distribution of cells along the crypt-axis, based on their crypt-axis score (Figure $1 E$ ), is altered in CD. We detected a significant shift in the density of cells toward the crypt top in CD relative to NIBD (Figure 2A). This is likely driven by specific subtypes of colonocytes, as we found that CA1+ colonocytes (Figure 2B), especially CA1+ late colonocytes (Figure $2 \mathrm{C}$ ), are significantly increased in abundance in $C D$ relative to NIBD. We observed only a slight difference in the relative abundance of mature absorptive cells (combined CA1+ early, CA1+ late, and CEACAM7+ colonocytes) vs. mature secretory cells (combined mature goblet cells and EECs) (Figure 2D). However, between the two major secretory lineages, we discovered a significant skew toward the mature goblet fate in $\mathrm{CD}$ (Figure $2 \mathrm{E}$ ). Even within the goblet lineage we observed a shift toward heightened goblet maturation in CD compared to NIBD (Figure 2F). 


\section{Different colonocyte clusters exhibit unique changes in gene expression in Crohn's disease}

To investigate the genes and pathways most altered in $C D$ in a cell-type specific manner, we first performed differential gene expression analysis in each of the 14 cell types separately. We identified a varying number of significantly (adjusted $P<0.05$ ) differentially expressed genes (DEGs) across cell types (Figure 3A). We found that the number of DEGs are roughly proportional to the number of cells in each of the clusters (Figure S2A), indicating that the variation in number of DEGs is due at least in part to variability in statistical power. A notable exception is the CA1+ late colonocyte cluster, which exhibits as many or more DEGs than several clusters with a greater number of cells, including the CA1+ early colonocyte, G2-M-G1 $\mathrm{TA}$, immature and mature goblet, and immature colonocyte clusters. Also, even though there are $>200$ fewer cells in the stem cell cluster compared to the mature goblet cell cluster, the stem cells exhibit more DEGs than mature goblet cells (Figure $3 \mathrm{~A}$ ), pointing to a robust change in the molecular profile of stem cells in CD. To investigate cell type specific changes in gene expression in the absorptive and secretory lineages, we first focused on two major colonocyte clusters (CA1+ late colonocyte and CEACAM7+ colonocyte) and two secretory clusters (mature goblet cells and EECs).

We found that 45 genes (30 up, 15 down in CD relative to NIBD) are significantly altered uniquely in $C A 1+$ late colonocytes (Table S2). Among those significantly up-regulated uniquely in $C A 1+$ late colonocytes are $P R D M 1$ and $R E L$, and among those down-regulated uniquely are CA2, SLC26A2, and SLC20A1 (Figure 3B). PRDM1 and REL have been implicated in antiinflammatory and microbial-sensing pathways in the colon, and have been reported as harboring variants associated with CD in genome-wide association studies (GWAS) (Ellinghaus et al., 2013; Khor et al., 2011); however, the increased expression in CD in only this subtype of colonocytes has not been demonstrated previously. CA2 and SLC26A2 are not only uniquely down-regulated in $C A 1+$ late colonocytes, but are also most highly expressed in this cluster (Figure $3 C$ ), suggesting that normal functions of this cell type such as anion transport are compromised in CD (SLC26A2 and SLC20A1 are sulfate and phosphate transporters, respectively, contributing to solute homeostasis in the colon).

There are 29 genes (7 up, 22 down) significantly altered uniquely in CEACAM7+ colonocytes (Table S3). Among those down-regulated are CA4, AQP8, GUCA2A, and GUCA2B (Figure $3 B$ ), each of which has been implicated in various normal functions of the colonic epithelium, such as the role of AQP8 in colonic epithelial water transport (Masyuk et al., 2002). Decreased colonic epithelial expression of AQP8 has been reported in IBD previously (Ricanek et al., 2015), and has been suggested as a candidate therapeutic target for diarrheal diseases (Escudero-Hernandez et al., 2020). Here, we show that the gene that codes for this important IBD-related protein is dramatically down-regulated in CD in CEACAM7+ colonocytes only and not in any other cell type of the colonic epithelium (Figure 3D).

We found that 4 genes ( 1 up, 3 down in CD relative to NIBD) are significantly altered in both CA1+ colonocytes and CEACAM7+ colonocytes, and in no other cell type. Shared down- 
regulated genes include $C D 177$ and $\angle Y P D 8$ (Figure 3B), both of which are in the same family of proteins containing the LY6/PLAUR domain. The latter encodes a protein that protects the gut from microbial invasion and is critical for maintaining barrier integrity and preventing intestinal inflammation (Hsu et al., 2017; Okumura et al., 2020; Okumura et al., 2016). LYPD8 is expressed 5 -fold greater in CEACAM7+ colonocytes compared to CA1+ late colonocytes, but it is significantly down-regulated in CD in both cell types (Figure 3E). Surprisingly, it is also highly expressed and dramatically reduced in CD in EECs (Figure 3E), though this decrease doesn't achieve significance likely due to the very small number of EECs, contributing to low statistical power.

The mature goblet program is enhanced whereas enteroendocrine drivers and L-cell markers are suppressed in Crohn's disease

Goblet cells secrete mucins to create a protective barrier for the colon from luminal content. In mature goblet cells, classic gene markers of maturity and function, including MUC2, MUC4, and TFF1 are significantly elevated in CD, whereas markers of immaturity, including $K L F 4, D L L 1$, and RETNLB, are significantly reduced in CD (Figure 3B). This is concordant with our finding of a shift toward heightened goblet maturation in CD compared to NIBD (Figure 2E). Interestingly, levels of CLDN4, previously reported to be highly expressed in EECs (Nagatake et al., 2014)and also studied in the context of colonocyte barrier function (Watari et al., 2017), are significantly elevated in the mature goblet cell cluster while modestly reduced in both CEACAM7+ colonocytes and EECs (Figure 3F). In fact, in CD, the levels of CLDN4 in mature goblet cells rise to what is observed in CEACAM7+ colonocytes (Figure 3F), the cells in which CLDN4 is most highly expressed in NIBD.

EECs, which secrete hormones in response to nutrients in order to maintain metabolic homeostasis, are not well-studied in CD. To examine the change in the molecular character of EECs in CD, we first examined the genes encoding key transcription factors $(n=10)$ that contribute to EEC maturation. We found that seven out of the ten are significantly altered in $C D$, all of which are down-regulated (Figure S3A), which is consistent with our observation that the abundance of EECs (relative to mature goblet) trends lower in CD compared to NIBD (Figure $2 \mathrm{E})$. We next evaluated the genes encoding the major hormones $(n=7)$ that are produced and secreted from EECs. We found that two in particular, GCG and PYY, both of which are expressed in the colonic L-cell subtype of EECs (Billing et al., 2018), are more than 3-fold reduced in CD vs. NIBD (Figure S3B). GCG encodes the key metabolic hormone GLP-1 that promotes systemic energy homeostasis (Barrera et al., 2011), and PYY encodes a signal that promotes satiety. Notably, the gene Nts, which encodes the pro-inflammatory peptide neurotensin (Castagliuolo et al., 1999), is highly up-regulated in CD (Figure S3B).

The canonical colonic stem cell signature is disrupted in Crohn's disease

Dysfunction in the intestinal stem cell population (ISC) in Crohn's disease has been proposed but not rigorously evaluated and documented. To investigate this possibility, we analyzed DEGs in the ISC population in CD relative to NIBD. We found that in ISCs, among the 
most highly up-regulated genes in CD are PLA2G2A and KLF6 (Figure 4A), both of which encode factors that are known to negatively regulate Wnt signaling in the crypts (Cheung et al., 2020; Schewe et al., 2016). Although we found that PLA2G2A is up-regulated in many other clusters also, KLF6 is significantly elevated primarily in ISCs. Accordingly, we observed that numerous genes in the Wnt signaling pathway (including CDCA7, CDK6, CCDC115, MYC, RNMT, TGIF1, $Y B X 1$, and FOS) are significantly reduced in expression in the ISC cluster in the CD samples relative to NIBD (Figure 4B). Moreover, in the case of CCDC115 and RNMT, they are significantly altered only in ISCs and not in any other cluster (Figure 4C).

We next assessed whether the Wnt-responsive, canonical marker of colonic stem cells, LGR5 (Barker et al., 2007), is affected in CD. We found that both the percentage of LGR5+ cells and the expression of $L G R 5$ in the ISC cluster are significantly reduced in CD relative to NIBD (Figure 4D). As a confirmation of this finding, we also detected a similar trend for SMOC2 (Figure 4E), which is known to be specifically enriched in the LGR5+ stem cell compartment (Munoz et al., 2012).

Intriguingly, we noticed that not all of the cells in the ISC cluster exhibit high expression of $L G R 5$ or SMOC2, even in the NIBD samples (Figure 4F). Previous work has distinguished three subtypes of ISCS: ISC-I, ISC-II, and ISC-III. ISC-I comprises canonical LGR5-high stem cells, whereas ISC-II and ISC-III are composed of LGR5-low stem cells that are more proliferative, more differentiated, and potentially antigen-presenting (Biton et al., 2018). We analyzed established markers (Biton et al., 2018) of the ISC-II and ISC-III subtypes, CD74 and LONP1, respectively, and confirmed that they are indeed present almost exclusively in the cells of the ISC cluster that are LGR5-low or LGR5-negative (Figure 4G). Moreover, we observed that both the percentage of $C D 74+$ cells and the expression of $C D 74$ in the ISC cluster are significantly increased in CD relative to NIBD (Figure $4 \mathrm{H}$ ). A similar trend was observed for the ISC-II marker gene LONP1 (Figure 4G,H). We also found that the vast majority of ISC-I marker genes (including but not limited to LGR5) are reduced in representation in CD ISCs (Figure 4I). Taken together, these data show that there is a shift away from an ISC-I signature in CD, indicative of a disrupted, non-homeostatic state in the colonic crypts.

\section{Detailed analysis of the SPIB+ cell cluster reveals new, rare cell types altered in Crohn's disease}

The least understood cell type that we have identified is the SPIB+ cluster. To dissect this further, we performed sub-clustering within this cluster only, and identified 4 sub-clusters (Figure 5A). We found that each of these sub-clusters expresses a unique set of markers (Methods, Figure 5B,C). Notably, the gene OTOP2 specifically marks SPIB+ sub-cluster 1; BEST4 (associated specifically with absorptive cells (Ito et al., 2013)) and CA7 are present only in SPIB+ sub-clusters 0 and 1; SLC12A2 is enriched in SPIB+ sub-clusters 2 and 3; and $L Y Z$ is elevated in $S P I B+$ sub-cluster 3 , though it is not highly specific (Figure $5 B, C$ ). The crypt-axis score analysis revealed a locational gradient of SPIB+ sub-clusters (Figure 5D). Only one of the sub-clusters (sub-cluster 1 ) is closer to the crypt-top, whereas the others are closer to crypt-bottom (Figure $5 D)$. Notably, we found that the relative abundance of $S P I B+$ sub-cluster $1(O T O P 2+/ C A 7+)$ is significantly elevated in CD as compared to NIBD (Figure 5E). 
We also noted that several markers of proliferation, particularly those that also mark the secretory progenitor (SP) cells, are enriched in SPIB+ sub-cluster 3 relative to the other $S P I B+$ sub-clusters. Among the 7 most specific markers of SP cells (Methods), we found that 4 (TOP2A, UBE2C, TPX2, and CENPF) are dramatically enriched in SPIB+ sub-cluster 3 relative to the other SPIB+ sub-clusters (Figure 5F). The remaining three, CLCA1, MUC2, and ITLN1, which are not found in $S P I B+$ sub-cluster 3, also mark immature and mature goblet cells (Figure 5F). Therefore, we labeled SPIB+ sub-cluster 3 as $L Y Z+$ non-goblet SP-like cells (Figure 5D). Notably, $L Y Z$ expression in these cells is significantly reduced in CD relative to NIBD (Figure $5 G$ ). We validated the presence of LYZ in crypt-bottom cells in NIBD samples and the depletion of this signal in $C D$ samples by immunohistochemistry (Figure $5 \mathrm{H}$ ). The lineage relationship among the $\mathrm{SPIB}+$ sub-clusters and other cluster is shown in Figure $5 \mathrm{I}$.

Genes implicated in Crohn's disease risk exhibit cell-type specific patterns of aberrant expression within the colonic epithelium

To investigate the colonic epithelial expression of genes implicated in $C D$, we first defined a list of genes $(n=261)$ nearest to every genetic variant significantly associated with $C D$ based on GWAS, of which 208 were detected in this dataset (Methods). For each of these genes we determined the percentage of cells positive and average expression across cells within each of the 14 colonic epithelial cell clusters (Figure 6A). We confirmed that genes expected to be enriched in the lamina propria, such as ILIO and NOD2, are not detected robustly in any of the epithelial cell clusters (Figure 6A). Indeed, several other genes fell into this category as well, including IL2RA, LTA, TNFSF8, CD244, and NELL1, suggesting that if these genes are involved in $C D$ pathophysiology, their primary roles are most likely outside of the epithelium. We observed that some $C D$ genes are detected at comparable levels across many epithelial cell clusters, such as ATG5, SKAP2, TAGLN2, PPM1G, and PRDX5, whereas other genes are enriched in only one or few cell types, such as ITLN1 (mature goblet cells) (Figure S4), CACNA2D1 (EECs), COL5A1 (EECS), RIPOR1 (EECS), IFNGR2 (CEACAM7+ colonocytes), NOTCH2 (SPIB+ cells), and ATG16L2 (SPIB+ cells). Investigating the latter two further within the SPIB+ cluster, we found that while NOTCH2 is ubiquitously expressed across all SPIB+ sub-clusters, ATG16L2 is enriched in SPIB+ sub-cluster 1 ( $L Y Z+$ non-goblet SP-like cells) (Figure 6B).

We next sought to determine whether any $C D$-associated genes are significantly altered in expression in CD relative to NIBD in one or more epithelial cell clusters. Some genes exhibit significant changes in gene expression across many clusters (Figure 7A), including HLA family genes such as HLA-DQB1, HLA-DRB1, and HLA-DRB5. These data point to the systematic increase in $\mathrm{CD}$ genes encoding for major histocompatibility complex (MHC) class II factors, generally active in antigen-presenting cells, across most epithelial cells including stem cells as described previously (Biton et al., 2018), with some minor exceptions (e.g., EECs for HLA-DRB5 or $C A 1+$ early colonocyte for $H L A-D Q B 1)$. Most genes that change in CD exhibit cell-type specific patterns. Notable examples include genes that are nearly specifically altered in EECs, including CACNA2D1, COL5A1, RIPOR1, RNF123, and LEMD2. The first three of these are more highly expressed in EECs in NIBD relative to other cell types (Figure 6A), whereas RNF123 and LEMD2 
expression levels are comparable across most cell types in NIBD but uniquely suppressed in EECs in CD patients (Figure 6A,7A).

Other examples include genes preferentially altered in stem cells, including $B A C H 2$, GPR65, and TNFRSF6B; in mature goblet cells, most notably ITLN1; as well as in SPIB+ cells, including FCHSD2 and NICN1. In the case of FCHSD2, it is suppressed across all SPIB+ subclusters, whereas the change in NICN1 is driven almost exclusively by SPIB+ sub-cluster 2 . We found that the CD gene JAK2 is also significantly elevated in SPIB+ cells (specifically, sub-cluster $3)$, though this aberration is shared with EECs as well (Figure 7A). The functions of FCHSD2 and NICN1 in CD pathophysiology are currently unknown, whereas JAK2 is well-studied in CD risk and JAK inhibitors are currently FDA-approved for IBD. Notably, though, the connection being made here between JAK2 and EECS or SPIB+ cells in the context of CD has not been reported previously.

\section{Discussion}

To our knowledge, this study represents the first single-cell analysis of colonic epithelial cells from treatment-naïve adult CD patients. A unique feature of this study is that the samples were isolated from treatment-naïve individuals, which means that the results are not confounded by the effects of drugs. Importantly, we specifically avoided macroscopically inflamed tissue in order to focus on cellular reprogramming that occurs more generally in the epithelial layer within the colon during CD. Inflammation can also lead to destruction of the epithelial layer, making it an unreliable indicator of IEC gene signatures. We identified almost 15 different types of epithelial cells, including several different subtypes of colonocytes and colonocyte progenitors. Each of the major clusters that express mature colonocyte markers, CEACAM7+ cells, CA1+ late colonocytes, and SPIB+ cells, express a unique set of genes. Notably, we found that CEACAM7+ cells are marked by LINCO1133, which as we understand is the first reported IncRNA marker of a colonic epithelial cell type. Although LINCO1133 has been implicated in the control of tumor phenotypes in colon cancer (Kong et al., 2016), its role in the normal function of CEACAM7+ colonocytes remains unknown and merits further investigation. Also, among the colonocyte clusters, the CA1+ late cluster is the only colonocyte subtype for which relative abundance is significantly altered in $C D$, and this too warrants study in the future.

Study of cellular composition revealed an increase in late-stage colonocytes in CD compared to healthy controls. However, in-depth analysis of the single cell data revealed aberrancies in the molecular character of these cells. For example, we observed dramatic reduction of solute and water transporters, suggestive of compromised colonocyte function in CD. Particularly notable is the dramatic decrease in AQP8 only in CEACAM7+ colonocytes. Although AQP8 has previously been shown to be suppressed in IBD (Ricanek et al., 2015), our study demonstrates for the first time that this effect is very likely driven by one specific colonocyte subtype. Furthermore, the significant reduction in CD of LYPD8, which encodes an important anti-microbial factor (Hsu et al., 2017; Okumura et al., 2020; Okumura et al., 2016), in both CEACAM7+ colonocytes and CA1+ late colonocytes, is a possible indication of the 
beginning stages of impaired colonocyte contribution to barrier function. We also show that LYPD8 is reduced in expression in EECs from CD patients, which has not been demonstrated previously.

Of note, we find that LYPD8 is modestly, albeit not significantly, increased in the mature goblet cell cluster. Also, mature goblet cells show both increased abundance in CD as well as increased expression of genes that code for mucins, including MUC2 and MUC4. This result is consistent with another recent study (Elmentaite et al., 2020), which reported a significant increase in goblet cells in pediatric ileal CD samples. This finding, coupled with alterations in the molecular phenotype of mature colonocytes, raises the possibility of a compensatory response of mature goblet cells to the potentially weakened function of colonocytes in CD. This notion is further supported by significantly increased CLDN4 expression in mature goblet cells in CD, which encodes for a tight-junction protein that promotes barrier integrity (Watari et al., 2017), to levels that match what is observed in CEACAM7+ colonocytes (where CLDN4 expression is normally most prominent).

We found a novel shift in ISCS away from the canonical LGR5+ signature in CD compared to NIBD, which motivates new avenues of future investigations into the dysfunction in ISCs as well as in lineage determination and colonic epithelial renewal during CD development. Among the numerous pro-Wnt signaling marker genes with reduced expression in ISCs in CD, two are uniquely suppressed only in ISCS, CCDC115 and RNMT. RNMT is recruited to Wnt signaling gene promoters by MYC (Posternak et al., 2017), which we show is suppressed in ISCs in CD, and therefore may be involved in mediating the shift away LGR5+ ISCs. To our knowledge, CCDC115 has not been studied previously in ISCS and may represent a novel factor in their function and $\mathrm{CD}$ etiology. We also found increased expression of MHC class II factors such as CD74, HLA$D Q B 1$, and HLA-DRB1, normally associated with antigen presentation, in ISCS of CD patients. A recent study showed that murine small intestinal stem cells act as non-conventional antigenpresenting cells to activate lamina propria T cells especially under certain conditions such as enteric infection (Biton et al., 2018). Whether this also occurs in CD is not known and merits further investigation.

One of the most intriguing findings in our study is that an initial cluster characterized by near ubiquitous SPIB, HES4, and NOTCH2 expression is more accurately annotated as four distinct sub-clusters based on unique markers that clearly distinguish each. Among these subclusters, the $O T O P 2+/ C A 7+$ sub-cluster, is significantly increased in relative abundance in CD compared to NIBD. Two previous single cell studies (Parikh et al., 2019; Smillie et al., 2019) of ulcerative colitis (UC) also identified this cell type but actually found that its abundance is decreased in UC patients compared to healthy controls. There are several possible explanations for this difference in our study: CD has a different etiology than UC; our analysis is focused on macroscopically non-inflamed regions; and our treatment-naïve patients may be in a different stage of disease course. These possibilities point to the importance of future single cell studies that carefully investigate differences between CD vs. UC and also include longitudinal assessments along disease course. We also identified a sub-cluster of SPIB+ cells, comprising $L Y Z+$ cells. Notably, $L Y Z$ expression is reduced and $L Y Z+$ cells are depleted in CD compared to 
NIBD, both at the RNA and protein level. The SPIB+/LYZ+ cells may correspond to previously annotated Paneth-like cells (PLCS)(Wang et al., 2020); however, besides $L Y Z$, we do not detect any classic markers of Paneth cells and therefore we termed them "non-goblet secretory progenitor-like cells" based on their gene signature.

Finally, in this study, we link CD GWAS risk genes to specific epithelial cell subtypes in which they are detected and/or aberrantly expressed. These discoveries may offer clues about the potential molecular mechanisms by which these genes contribute to CD etiology. For example, ATG16L2 is enriched in the SPIB+ cluster, particularly within the $S P I B+/ L Y Z+$ subcluster, suggesting that this gene may contribute to $C D$ etiology through the novel functions of this uncharacterized cluster, which may center on anti-microbial functions given the presence of $L Y Z$. We also found that increased JAK2 expression is prominent not only in the SPIB+ cluster but also in EECs, which is completely unexpected and merits more detailed functional investigation given that JAK inhibitors are approved for use in ulcerative colitis and are in clinical trials for CD. Overall, we believe that this study offers a unique picture, at unprecedented resolution, of the cellular and molecular landscape of the colonic epithelium in treatment-naïve adult CD.

Future single-cell studies must expand on this work to include larger cohorts and to assess the impact of age, gender, CD sub-type (Keith et al., 2018; Weiser et al., 2018), disease region (e.g., ileum vs. colon), disease duration, and treatment history on cell composition and molecular phenotype. Longitudinal investigations to uncover changes to the cellular landscape and molecular phenotype over the course of disease progression are also merited. Finally, it will be exciting in the future to explore the relationship between specific luminal bacterial changes and alterations at the single-cell level, given the intimate relationship between microbial dysbiosis and CD pathogenesis.

\section{Methods}

\section{Ethical statement}

The study was conducted in accordance with the Declaration of Helsinki and Good Clinical Practice. The study protocol was approved by the institutional review board at University of North Carolina at Chapel Hill (approval number: 19-0819 and 17-0236). All participants provided written informed consent prior to inclusion in the study. All participants are identified by number and not by name or any protected health information.

\section{Single cell RNA-sequencing}

Colonic mucosa was obtained endoscopically as biopsies from patients with treatment-naive CD and NIBD healthy controls. Cross-sectional clinical data was collected the time of sampling. All samples were collected from regions of ascending colon without macroscopic inflammation. Isolation of colonic primary IECs was performed as reported previously (Toyonaga et al., 2020; Wang et al., 2017). This method has previously been shown to result in $>95 \%$ purity of IECs 
(Camp et al., 2014). Single cell libraries were constructed using the Chromium Single Cell 3'Reagent Kits (V3) according to manufacturer instructions.

\section{Single cell transcriptome data analysis}

Sample single cell fastqs were aligned to the human genome (GRCh38-3.1.0) using 10x Genomics cellranger count (v4.0.0) to obtain gene/cell count matrices. Sample filtering, normalization, integration, clustering and visualization was accomplished using Seurat (v3.2). To maintain sample quality, cells with less than 1000 detected genes or greater than $25 \%$ of reads aligning to mitochondrial genes were removed. Additionally, cells with greater than 50000 reads were removed as potential doublets. To control for read depth, sample counts were log normalized. Samples were merged using the Seurat integration anchor workflow based on the 2000 most variable genes. Clustering and identification of nearest neighbors relied on 11 PCA dimensions. Cells were clustered at a resolution of 0.7 . To focus on epithelial cells, immune clusters were identified by expression of known immune cell type markers. Immune clusters were removed, and the integration workflow was repeated using the 2000 most variable genes of the remaining epithelial cells. Clustering and identification of nearest neighbors relied on 10 PCA dimensions. Epithelial cells were clustered using a resolution of 0.8 . Highly enriched markers for each cluster were determined using the Seurat function FindAllMarkers, which compares the gene expression within a cluster to all other clusters. Genes that are up-regulated with a log fold change greater than 0.25 and a Wilcoxon Rank Sum test $p$-value $<0.01$ were considered highly enriched markers. Clusters were assigned cell types based on expression of known markers and/or highly enriched markers of the cluster. Despite earlier filtering, one of the resulting clusters was immune cells and therefore removed from the analysis. Samples were found to have similar count, genes, and mitochondrial read percentage per cell (Table S4). Differential expression of genes between CD and NIBD samples was determined within clusters using the Wilcoxon Rank Sum test.

Sub-clustering of SPIB+ cells was performed to identify discrete cell types within the cluster. The SPIB+ cells were sub-clustered using the integrated counts. Clustering and identification of nearest neighbors relied on 10 PCA dimensions. SPIB+ cells were sub-clustered using a resolution of 0.8 . Assignment of cell types to sub-clusters was determined by the highly enriched markers of the subcluster.

\section{Determination of highly enriched marker genes}

Highly enriched genes were determined by one of two different thresholds. In both thresholds, the genes had to be up-regulated $>0.5$ log fold change in the cluster of interest vs. all other clusters. Using the stringent threshold, a gene had to be expressed in greater than $90 \%$ of the cells in the cluster of interest and less than $30 \%$ in all other clusters (Figure 5F). Using the slightly relaxed threshold, a gene had to be expressed in greater than $80 \%$ of cells in the cluster of interest and less than $40 \%$ of cells in all other clusters (Figure 1D). 
The crypt-axis score was assigned for each cell and was based on the expression of a previously defined set of genes: SELENOP, CEACAM7, PLAC8, CEACAM1, TSPAN1, CEACAM5, CEACAM6, IFI27, DHRS9, KRT20, RHOC, CD177, PKIB, HPGD, LYPD8 (Parikh et al., 2019). For each gene, expression within a cell was divided by the max expression across all cells to mitigate the weight of highly expressed genes. The max expression normalized values places the gene's expression on a 0 to 1 scale. The crypt-axis score is the summation across all genes of the maxnormalized expression values.

\section{Intestinal stem cell analysis}

Genes constituting the three classes of intestinal stem cells (ISC) were obtained from Biton et al (Biton et al., 2018). In the stem cluster, the percent of NIBD or CD cells positive for the genes was calculated and the delta was determined by subtracting the NIBD percent positive from the CD percent positive for each gene. The overall shift in an ISC class was determined by averaging the delta across all genes within the ISC class.

\section{Integrative analysis with IBD genome-wide association study results}

A list of 5743 Crohn's disease risk-associated SNPs were identified through GWAS. The closest genes to the disease risk-associated SNPs were identified using bedtools closest (v2.27), resulting in 261 unique genes. Of these genes, 208 were present in the filtered dataset. For the figures, the gene order was determined by hierarchical clustering of the Euclidean distance of the log2 fold change for each gene across clusters.

\section{Velocyto}

The velocity pseudotime figure was generated using scVelo (v0.2.2). The velocities were computed using the dynamical model, and we performed a likelihood-ratio test to test for differential kinetics between clusters and corrected the velocity for differential kinetics. The root index was set as the first indexed stem cell.

\section{PAGA}

The trajectory inference graph was generated using PAGA, which was implemented in Scanpy (v1.6). Pruning was done by setting a minimum edge weight of 0.3. Additionally, the tree was rooted at the stem cluster.

\section{Histological analysis}

Human proximal colonic tissue was fixed in $4 \%(\mathrm{v} / \mathrm{v})$ neutral-buffered paraformaldehyde, embedded in paraffin, and cut into $10 \mu \mathrm{m}$ sections. Immunofluorescent staining of LYZ was performed to visualize Paneth cells. Briefly, sections were incubated with primary antibody (rabbit anti-LYZ, 1:1000 dilution in PBS with 1\% (w/v) BSA) (Invitrogen, Carlsbad, CA, cat. PA5- 
16668) overnight at $4^{\circ} \mathrm{C}$ followed by goat anti-rabbit Alexa fluor 594 secondary antibody (1:1000 in PBS with 1\% (w/v) BSA) (Invitrogen, Carlsbad, CA, cat. A1102) incubation for $1 \mathrm{hr}$ at room temperature. Subsequent section incubation with DAPI (1:1000 in PBS) (Invitrogen, Carlsbad, CA, cat. D1306) for $30 \mathrm{~min}$. at room temperature was used to visualize nuclei. Images were captured using a BX53 Olympus scope (Olympus, Center Valley, PA). LYZ+ cells were enumerated in longitudinally well-oriented colonic crypts.

\section{Acknowledgments}

This work was funded in part through Helmsley Charitable Trust (SHARE Project 2), NIDDK P01DK094779, NIDDK 1R01DK104828-01A1, NIDDK P30-DK034987, NIH T32 Translational Medicine Training Grant (T32-GM122741), and Research Fellow Award from Crohn's and Colitis Foundation. The UNC Translational Pathology Laboratory is supported, in part, by grants from the National Cancer Institute (3P30CA016086).

Figure Legends

Figure 1: Single cell landscape in treatment-naïve Crohn's disease and NIBD patients (A) UMAP of epithelial cell clusters following assignment of cell type. (B) Normalized expression heatmap of genes known to be markers of various epithelial cell populations or found to be highly enriched in a cluster (rows) across epithelial clusters (columns) confirms cell type assignment. Normalized gene expression is scaled by row. (C) UMAP overlain with the normalized expression of cell type markers further confirms cluster assignment. MUC2 = goblet, LGR5 = stem, CA1 = CA1+ colonocytes, CEACAM7 = CEACAM7+ colonocytes, SPIB = SPIB+ cells, CENPA = G2-M-G1 TA, PCNA = S phase TA, CHGA = EEC. (D) Normalized expression heatmap of three clusters with a colonocytic signature (CA1+ late colonocytes, CEACAM7+ colonocytes, and $\mathrm{SPIB}+$ cells) reveal expression of cluster-specific markers. Normalized expression is scaled by row. (E) Crypt-axis scores (low near crypt bottom, high near crypt top) of cells reveals expected location of clusters along the crypt axis. Clusters are arranged on $\mathrm{x}$-axis by mean crypt-axis score (Methods). The size of the dot corresponds to level of expression of known marker of the crypt top, SELENOP. Diagram of colonic crypt shown at right. (F) UMAP overlain with the RNA velocity. RNA velocity is the ratio of spliced transcripts / unspliced transcripts. Scale is normalized to 1 . NIBD = Non-inflammatory bowel disease, $C D=$ Crohn's disease, UMAP = Uniform Manifold Approximation and Projection, EEC = enteroendocrine cells.

Figure 2: Crohn's disease causes shifts in colonic epithelial landscape

(A) Crypt-axis score density for NIBD and CD cells separately reveals a shift toward the colonic crypt top in CD. Significance of shift was determined using Kolmogorov-Smirnov test $(p<0.001$ $=* * *)(B)$ Mean cell abundances across NIBD and CD samples reveals significant increase in CA1+ colonocytes in CD. Dots show abundance values of individual samples. (C) Mean cell abundances for either CA1+ early or late colonocyte clusters across NIBD and CD samples reveals a significant increase only in the $\mathrm{CA} 1+$ late colonocytes. Dots show abundances of individual samples. Abundances of other clusters not shown. (D) Mean ratio of absorptive to secretory cells across NIBD and CD samples. (E) Mean ratio of EEC to mature goblet cells across 
NIBD and CD samples reveals a significant shift in the secretory lineage toward mature goblet cells in CD. (F) Mean ratio of immature goblet to mature goblet across NIBD and CD samples reveal a shift toward mature goblet cells in CD. If not indicated, $p$-values are calculated by a Student's t-test $(\mathrm{p}<0.05=*, \mathrm{p}<0.01=* *)$. NIBD $=$ Non-inflammatory bowel disease, $\mathrm{CD}=$ Crohn's disease, EEC = enteroendocrine cells.

\section{Figure 3: Effect of Crohn's disease within cell types}

(A) Number of significant differentially expressed genes in CD versus NIBD. Clusters are shown on the $x$-axis followed by the number of cells within the cluster in parenthetical notation and the number of differentially expressed genes is shown on the y-axis. (B) Differentially expressed genes for three clusters; CA1+ late colonocyte, CEACAM7+ colonocyte, and mature goblet. Log2 fold change is shown on $\mathrm{x}$-axis and adjusted $\mathrm{p}$-value is shown on the $\mathrm{y}$-axis. (C-F) Mean expression of SLC26A2 \& CA2 (C), AQP8 (D), CD177 \& LYPD8 (E), and CLDN4 (F) across clusters for NIBD and CD samples demonstrates the specificity of expression and differential expression within clusters. P-values are calculated by Wilcoxon Rank Sum test (adjusted $p<0.05=*$, adjusted $\mathrm{p}<0.01=* *$, adjusted $\mathrm{p}<0.001=* * *)$. NIBD $=$ Non-inflammatory bowel disease, $\mathrm{CD}=$ Crohn's disease.

\section{Figure 4: Crohn's disease disrupts stem cell homeostasis}

(A) Differentially expressed gene within the stem cluster. Log2 fold change is shown on x-axis and adjusted $p$-value on the $y$-axis. (B) Mean expression of eight mediators of the Wnt pathway in NIBD and CD samples reveal a consistent decrease in response to CD. (C) Mean expression of $C C D C 115$ \& RNMT across clusters shows a significant decrease only occurs in the stem cluster. (D-E) Percent of cells positive for $L G R 5$ (D, left) and SMOC2 (E, left) or the average normalized expression for $L G R 5$ (D, right) and SMOC2 (E, right) in stem cluster for NIBD or CD samples. Dots relate to individual sample value. (F) UMAP of the stem cluster with expression of $L G R 5$ or SMOC2 overlain, separated by NIBD/CD. (G) UMAP of stem cluster with LGR5 expression (red) co-overlain with either CD74 (top) or LONP1 (bottom) (both blue) expression. Scale for expression is shown on right. $(\mathrm{H})$ Percent of cells positive for CD74 (left top) and LONP1 (left bottom) or the average normalized expression for CD74 (right top) and LONP1 (right bottom) in stem cluster for NIBD or CD samples. Dots relate to individual sample value. (I) Shift in percent positive of genes in either ISC-I, ISC-II, or ISC-III cell types between NIBD and CD. P-values for expression bar plots was calculated using the Wilcoxon Rank Sum test and the $p$-value for the ISC subtype shifts was calculated using a Student's t-test $(\mathrm{p}<0.05=*, \mathrm{p}<0.01=* *, \mathrm{p}<0.001=$ $* * *)$. NIBD = Non-inflammatory bowel disease, CD = Crohn's disease, ISC = intestinal stem cell, UMAP = Uniform Manifold Approximation and Projection.

\section{Figure 5: Subcluster analysis of SPIB+ cells reveals distinct lineages}

(A) UMAP following subclustering of SPIB+ cells with labeling of the four identified subclusters.

(B) UMAP of SPIB + cell subclusters overlain with gene expression from genes marking the entire cluster or distinct subclusters. (C) SPIB+ cell subcluster UMAP following cell type assignment using highly enriched markers of the subclusters. (D) Crypt-axis scores (low near crypt bottom, high near crypt top) of subcluster-assigned cells uncovers a colonocyte signature in the OTOP2+/CA7+ subcluster. Clusters are arranged on $x$-axis by mean crypt-axis score. The size of 
the dot corresponds to level of expression of SELENOP, a known marker of the top of the crypt. (E) Mean cluster cell abundances across NIBD and CD samples reveals significant increase in OTOP2+/CA7+ subcluster in CD. Dots show abundances of individual samples. (F) UMAP of either all clusters (top) or SPIB+ cell subclusters (bottom) overlain with the expression of markers of secretory progenitor cells. (G) Mean normalized expression of $L Y Z$ in NIBD and CD samples in the LYZ+ non-goblet SP-like subcluster. $(\mathrm{H}) \mathrm{LYZ}$-detected immunofluorescence in colonic crypts from either NIBD or CD patients examples (left) and quantified by sample (right). Mean percent of crypts positive for LYZ in NIBD or CD. (I) Lineage reconstruction as determined by PAGA. NIBD = Non-inflammatory bowel disease, $C D=$ Crohn's disease, UMAP = Uniform Manifold Approximation and Projection, PAGA = partition-based graph abstraction.

Figure 6: Expression of CD-associated risk genes across clusters

(A) Dot plots showing expression and percentage of cells expressing CD-associated risk genes (columns) across clusters (rows). For each gene, color of dot corresponds to the average scaled expression and the size of the dot corresponds to the percent of cells in cluster expressing the gene. (B) UMAP of SPIB+ cells subclusters overlain with gene expression from NOTCH2 or ATG16L2. NIBD = Non-inflammatory bowel disease, $C D=$ Crohn's disease, UMAP = Uniform Manifold Approximation and Projection.

Figure 7: Differential expression of CD-associated risk genes across clusters (A) Dot plots showing the log2 fold change of CD compared to NIBD within clusters, the direction of change, and the statistical significance of CD-associated risk genes (columns) across clusters (rows). For each gene, color of the dot corresponds to the log 2 fold change, the shape of the dot corresponds to the direction of change, and the color of the dot corresponds to the adjusted $p$-value of the comparison. Adjusted $p$-value was calculated from the Wilcoxon Rank Sum test. NIBD = Non-inflammatory bowel disease, $C D=$ Crohn's disease.

\section{Supplemental Figure 1: Clustering and identification of contaminating immune cells} (A) UMAP of initial clusters (left) and clusters following cell type annotation using known cell type markers and highly enriched genes (right). (B) Violin plots showing normalized expression of known markers of different immune cell types on the $y$-axis and the immune clusters on the $\mathrm{x}$-axis. UMAP = Uniform Manifold Approximation and Projection.

\section{Supplemental Figure 2: Correlation between cluster size and identification of significant differentially expressed genes}

(A) The number of significant differentially expressed genes (x-axis) increases with number of cells in cluster ( $y$-axis). Dashed line is the linear model with the corresponding $R$ value shown in upper quadrant of plot.

\section{Supplemental Figure 3: CD disrupts EEC homeostatsis}

(A) Mean normalized expression across 10 transcription factors involved in EEC maturation in NIBD and CD samples. (B) Mean normalized expression across 7 EEC hormones in NIBD and CD samples. P-values were calculated using the Wilcoxon Rank Sum test $(p<0.05=*, p<0.01=* *$, 
$\mathrm{p}<0.001=* * *)$. NIBD $=$ Non-inflammatory bowel disease, $\mathrm{CD}=$ Crohn's disease, $\mathrm{EEC}=$ Enteroendocrine cells.

\section{Supplemental Figure 4: Expression of ITLN1 in mature goblet cells}

(A) UMAP of all epithelial clusters (top left) or the mature goblet cluster (top right) overlain with ITLN1 expression. UMAP of all epithelial clusters overlain with both MUC2 (blue) and ITLN1 (red) expression (bottom).

Supplemental Table 1: Information on the seven individuals from which the single cell tissue was biopsied.

Supplemental Table 2: Significant ( $p$-adjusted < 0.05) differentially expressed genes in CD compared to NIBD that are specific to CA1+ late colonocytes.

Supplemental Table 3: Significant ( $p$-adjusted $<0.05$ ) differentially expressed genes in CD compared to NIBD that are specific to CEACAM7+ colonocytes.

Supplemental Table 4: Average UMI count, detected genes, and percent of mitochondrial genes per cell across the seven samples used. 


\section{References}

Barker, N., van Es, J.H., Kuipers, J., Kujala, P., van den Born, M., Cozijnsen, M., Haegebarth, A., Korving, J., Begthel, H., Peters, P.J., et al. (2007). Identification of stem cells in small intestine and colon by marker gene Lgr5. Nature 449, 1003-1007.

Barrera, J.G., Sandoval, D.A., D'Alessio, D.A., and Seeley, R.J. (2011). GLP-1 and energy balance: an integrated model of short-term and long-term control. Nat Rev Endocrinol 7, 507-516.

Becht, E., McInnes, L., Healy, J., Dutertre, C.A., Kwok, I.W.H., Ng, L.G., Ginhoux, F., and Newell, E.W. (2018). Dimensionality reduction for visualizing single-cell data using UMAP. Nat Biotechnol.

Billing, L.J., Smith, C.A., Larraufie, P., Goldspink, D.A., Galvin, S., Kay, R.G., Howe, J.D., Walker, R., Pruna, M., Glass, L., et al. (2018). Co-storage and release of insulin-like peptide-5, glucagonlike peptide- 1 and peptideYY from murine and human colonic enteroendocrine cells. Mol Metab 16, 65-75.

Biton, M., Haber, A.L., Rogel, N., Burgin, G., Beyaz, S., Schnell, A., Ashenberg, O., Su, C.W., Smillie, C., Shekhar, K., et al. (2018). T Helper Cell Cytokines Modulate Intestinal Stem Cell Renewal and Differentiation. Cell 175, 1307-1320 e1322.

Camp, J.G., Frank, C.L., Lickwar, C.R., Guturu, H., Rube, T., Wenger, A.M., Chen, J., Bejerano, G., Crawford, G.E., and Rawls, J.F. (2014). Microbiota modulate transcription in the intestinal epithelium without remodeling the accessible chromatin landscape. Genome Res 24, 15041516.

Castagliuolo, I., Wang, C.C., Valenick, L., Pasha, A., Nikulasson, S., Carraway, R.E., and Pothoulakis, C. (1999). Neurotensin is a proinflammatory neuropeptide in colonic inflammation. J Clin Invest 103, 843-849.

Cheung, P., Xiol, J., Dill, M.T., Yuan, W.C., Panero, R., Roper, J., Osorio, F.G., Maglic, D., Li, Q., Gurung, B., et al. (2020). Regenerative Reprogramming of the Intestinal Stem Cell State via Hippo Signaling Suppresses Metastatic Colorectal Cancer. Cell Stem Cell 27, 590-604 e599. Ellinghaus, D., Zhang, H., Zeissig, S., Lipinski, S., Till, A., Jiang, T., Stade, B., Bromberg, Y., Ellinghaus, E., Keller, A., et al. (2013). Association between variants of PRDM1 and NDP52 and Crohn's disease, based on exome sequencing and functional studies. Gastroenterology 145, 339-347.

Elmentaite, R., Ross, A.D.B., Roberts, K., James, K.R., Ortmann, D., Gomes, T., Nayak, K., Tuck, L., Pritchard, S., Bayraktar, O.A., et al. (2020). Single-Cell Sequencing of Developing Human Gut Reveals Transcriptional Links to Childhood Crohn's Disease. Dev Cell 55, 771-783 e775.

Escudero-Hernandez, C., Munch, A., Ostvik, A.E., Granlund, A.V.B., and Koch, S. (2020). The Water Channel Aquaporin 8 is a Critical Regulator of Intestinal Fluid Homeostasis in Collagenous Colitis. J Crohns Colitis 14, 962-973.

Hsu, C.C., Okumura, R., and Takeda, K. (2017). Human LYPD8 protein inhibits motility of flagellated bacteria. Inflamm Regen 37, 23.

Ito, G., Okamoto, R., Murano, T., Shimizu, H., Fujii, S., Nakata, T., Mizutani, T., Yui, S., AkiyamaMorio, J., Nemoto, Y., et al. (2013). Lineage-specific expression of bestrophin-2 and bestrophin4 in human intestinal epithelial cells. PLoS One 8, e79693. 
Keith, B.P., Barrow, J.B., Toyonaga, T., Kazgan, N., O'Connor, M.H., Shah, N.D., Schaner, M.S., Wolber, E.A., Trad, O.K., Gipson, G.R., et al. (2018). Colonic epithelial miR-31 associates with the development of Crohn's phenotypes. JCl Insight 3.

Khor, B., Gardet, A., and Xavier, R.J. (2011). Genetics and pathogenesis of inflammatory bowel disease. Nature 474, 307-317.

Kong, J., Sun, W., Li, C., Wan, L., Wang, S., Wu, Y., Xu, E., Zhang, H., and Lai, M. (2016). Long non-coding RNA LINC01133 inhibits epithelial-mesenchymal transition and metastasis in colorectal cancer by interacting with SRSF6. Cancer Lett 380, 476-484.

La Manno, G., Soldatov, R., Zeisel, A., Braun, E., Hochgerner, H., Petukhov, V., Lidschreiber, K., Kastriti, M.E., Lonnerberg, P., Furlan, A., et al. (2018). RNA velocity of single cells. Nature 560, 494-498.

Masyuk, A.I., Marinelli, R.A., and LaRusso, N.F. (2002). Water transport by epithelia of the digestive tract. Gastroenterology 122, 545-562.

Munoz, J., Stange, D.E., Schepers, A.G., van de Wetering, M., Koo, B.K., Itzkovitz, S., Volckmann, R., Kung, K.S., Koster, J., Radulescu, S., et al. (2012). The Lgr5 intestinal stem cell signature: robust expression of proposed quiescent '+4' cell markers. EMBO J 31, 3079-3091.

Nagatake, T., Fujita, H., Minato, N., and Hamazaki, Y. (2014). Enteroendocrine cells are specifically marked by cell surface expression of claudin-4 in mouse small intestine. PLoS One 9, e90638.

Okumura, R., Kodama, T., Hsu, C.C., Sahlgren, B.H., Hamano, S., Kurakawa, T., lida, T., and

Takeda, K. (2020). Lypd8 inhibits attachment of pathogenic bacteria to colonic epithelia.

Mucosal Immunol 13, 75-85.

Okumura, R., Kurakawa, T., Nakano, T., Kayama, H., Kinoshita, M., Motooka, D., Gotoh, K., Kimura, T., Kamiyama, N., Kusu, T., et al. (2016). Lypd8 promotes the segregation of flagellated microbiota and colonic epithelia. Nature 532, 117-121.

Parikh, K., Antanaviciute, A., Fawkner-Corbett, D., Jagielowicz, M., Aulicino, A., Lagerholm, C., Davis, S., Kinchen, J., Chen, H.H., Alham, N.K., et al. (2019). Colonic epithelial cell diversity in health and inflammatory bowel disease. Nature 567, 49-55.

Posternak, V., Ung, M.H., Cheng, C., and Cole, M.D. (2017). MYC Mediates mRNA Cap

Methylation of Canonical Wnt/beta-Catenin Signaling Transcripts By Recruiting CDK7 and RNA Methyltransferase. Mol Cancer Res 15, 213-224.

Ricanek, P., Lunde, L.K., Frye, S.A., Stoen, M., Nygard, S., Morth, J.P., Rydning, A., Vatn, M.H., Amiry-Moghaddam, M., and Tonjum, T. (2015). Reduced expression of aquaporins in human intestinal mucosa in early stage inflammatory bowel disease. Clin Exp Gastroenterol 8, 49-67. Schewe, M., Franken, P.F., Sacchetti, A., Schmitt, M., Joosten, R., Bottcher, R., van Royen, M.E., Jeammet, L., Payre, C., Scott, P.M., et al. (2016). Secreted Phospholipases A2 Are Intestinal Stem Cell Niche Factors with Distinct Roles in Homeostasis, Inflammation, and Cancer. Cell Stem Cell 19, 38-51.

Smillie, C.S., Biton, M., Ordovas-Montanes, J., Sullivan, K.M., Burgin, G., Graham, D.B., Herbst, R.H., Rogel, N., Slyper, M., Waldman, J., et al. (2019). Intra- and Inter-cellular Rewiring of the Human Colon during Ulcerative Colitis. Cell 178, 714-730 e722.

Toyonaga, T., Steinbach, E.C., Keith, B.P., Barrow, J.B., Schaner, M.R., Wolber, E.A., Beasley, C., Huling, J., Wang, Y., Allbritton, N.L., et al. (2020). Decreased Colonic Activin Receptor-Like 
Kinase 1 Disrupts Epithelial Barrier Integrity in Patients With Crohn's Disease. Cell Mol Gastroenterol Hepatol 10, 779-796.

Wang, Y., DiSalvo, M., Gunasekara, D.B., Dutton, J., Proctor, A., Lebhar, M.S., Williamson, I.A., Speer, J., Howard, R.L., Smiddy, N.M., et al. (2017). Self-renewing Monolayer of Primary Colonic or Rectal Epithelial Cells. Cell Mol Gastroenterol Hepatol 4, 165-182 e167.

Wang, Y., Song, W., Wang, J., Wang, T., Xiong, X., Qi, Z., Fu, W., Yang, X., and Chen, Y.G. (2020). Single-cell transcriptome analysis reveals differential nutrient absorption functions in human intestine. J Exp Med 217.

Watari, A., Kodaka, M., Matsuhisa, K., Sakamoto, Y., Hisaie, K., Kawashita, N., Takagi, T., Yamagishi, Y., Suzuki, H., Tsujino, H., et al. (2017). Identification of claudin-4 binder that attenuates tight junction barrier function by TR-FRET-based screening assay. Sci Rep 7, 14514. Weiser, M., Simon, J.M., Kochar, B., Tovar, A., Israel, J.W., Robinson, A., Gipson, G.R., Schaner, M.S., Herfarth, H.H., Sartor, R.B., et al. (2018). Molecular classification of Crohn's disease reveals two clinically relevant subtypes. Gut 67, 36-42.

Yang, X.Z., Cheng, T.T., He, Q.J., Lei, Z.Y., Chi, J., Tang, Z., Liao, Q.X., Zhang, H., Zeng, L.S., and Cui, S.Z. (2018). LINC01133 as ceRNA inhibits gastric cancer progression by sponging miR-106a$3 p$ to regulate APC expression and the Wnt/beta-catenin pathway. Mol Cancer 17, 126. 


\section{Figure 1}

A

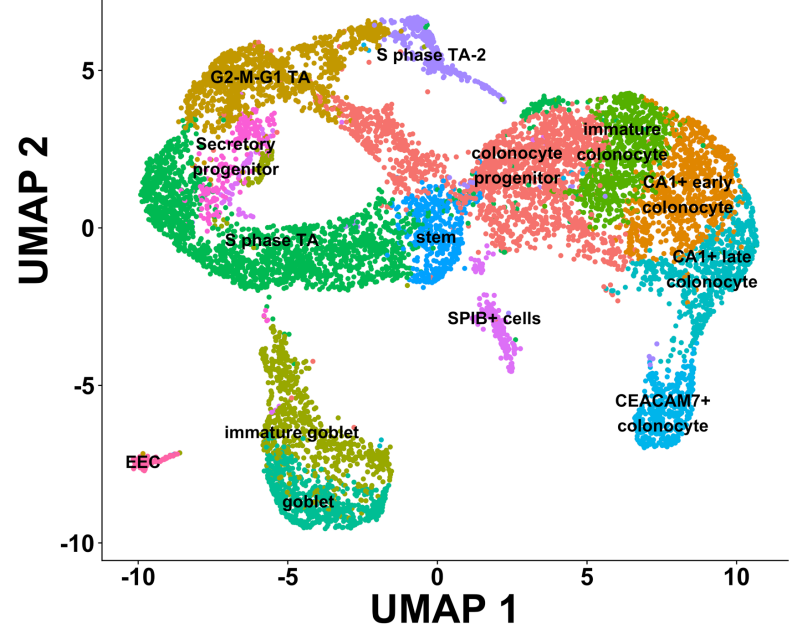

B

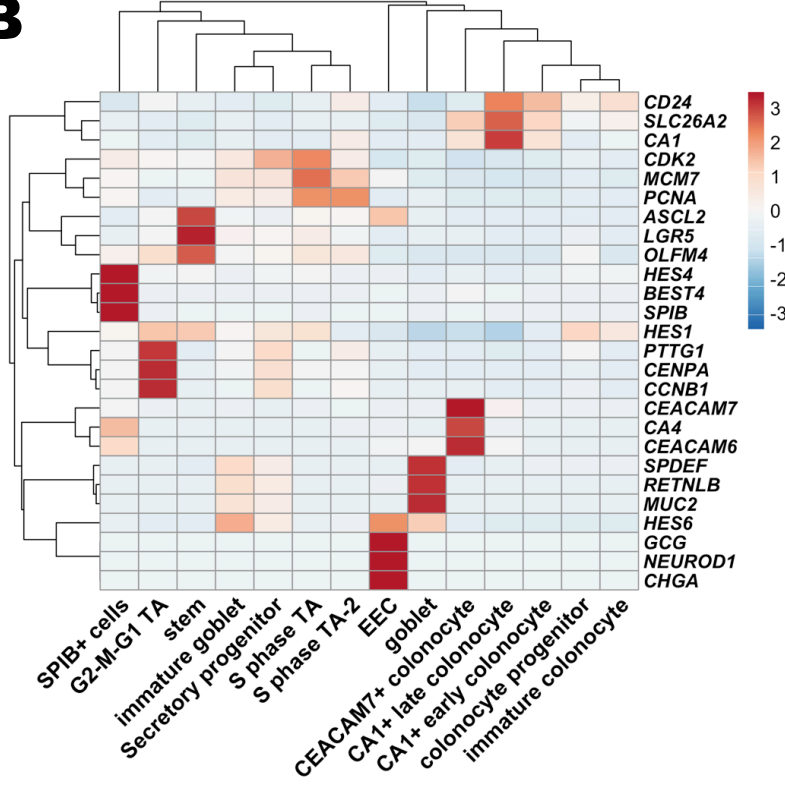

C
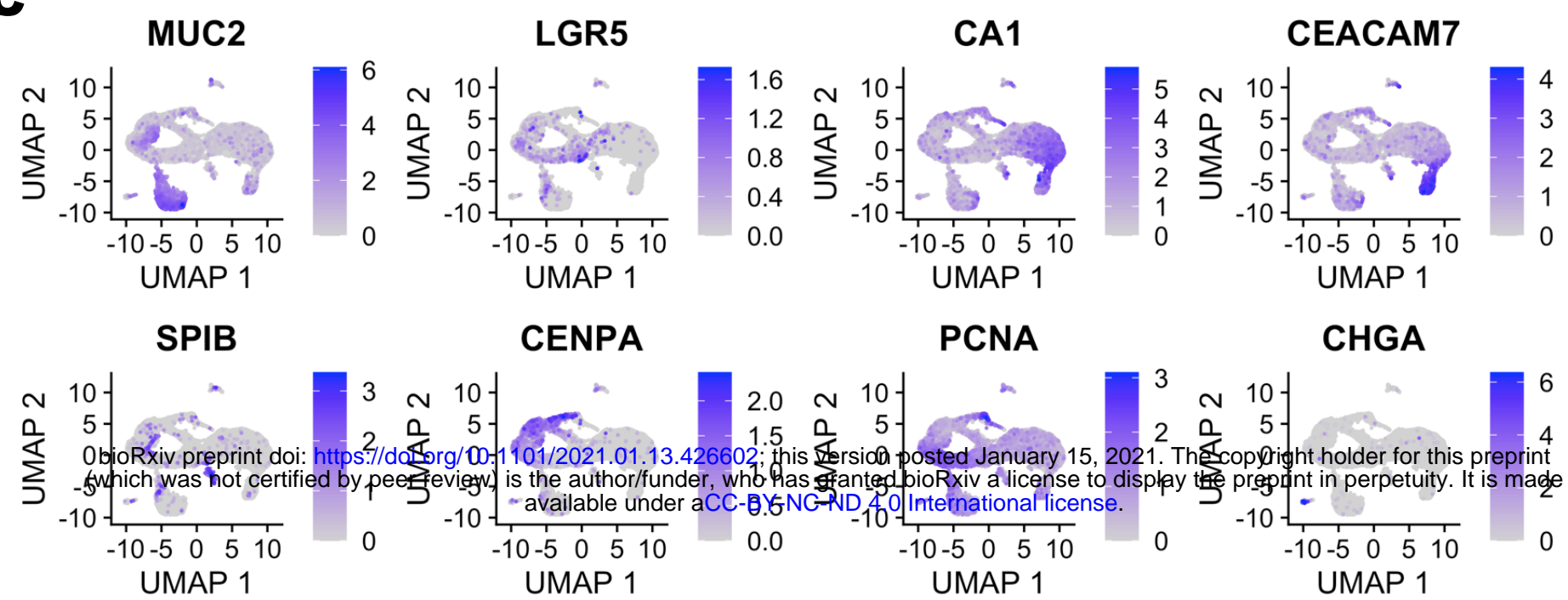

D

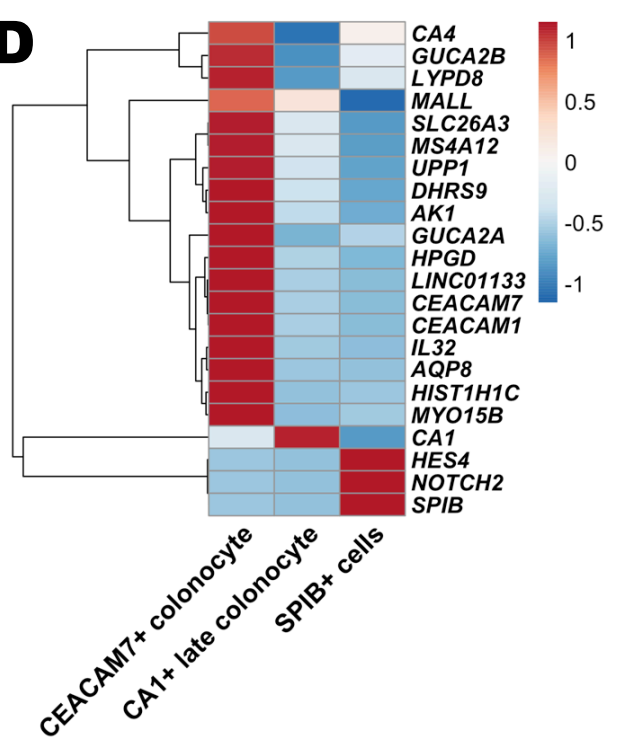

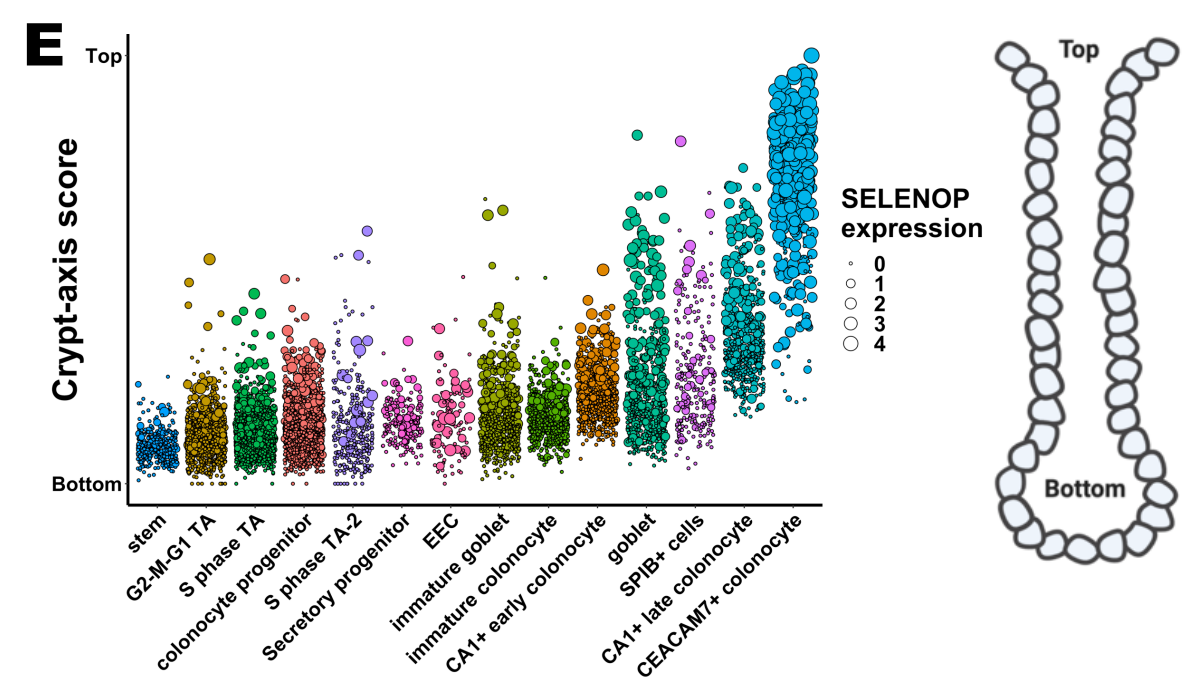

F

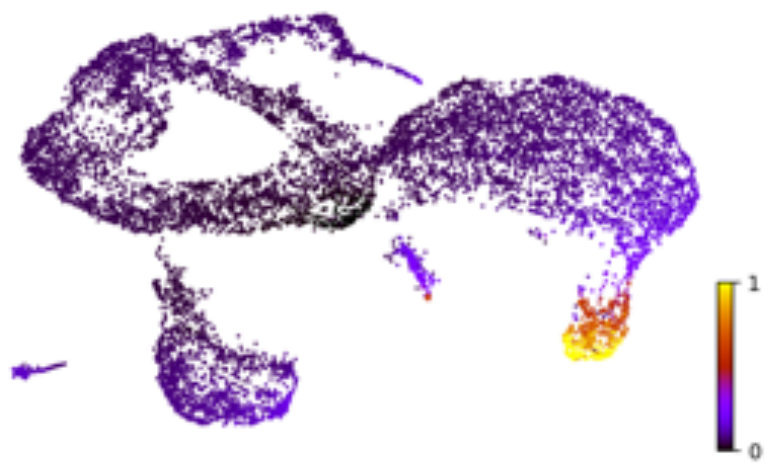


Figure 2

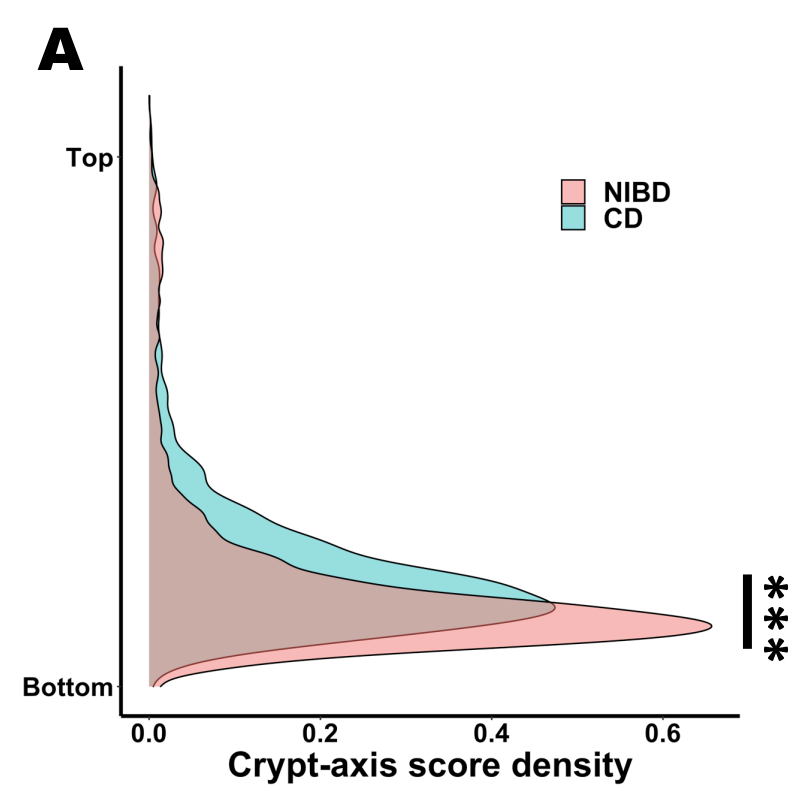

C

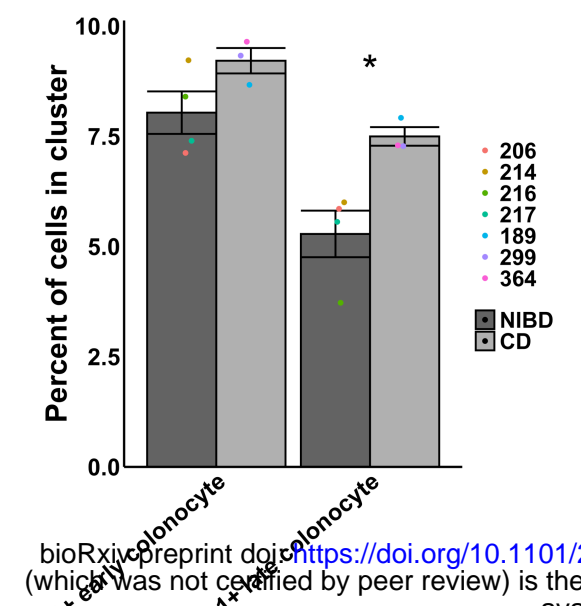

2021.01.13.426602; this version pefted January 15, 2021. The cppyright holder fol this preprint

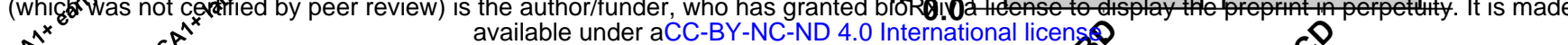

$\mathbf{E}$

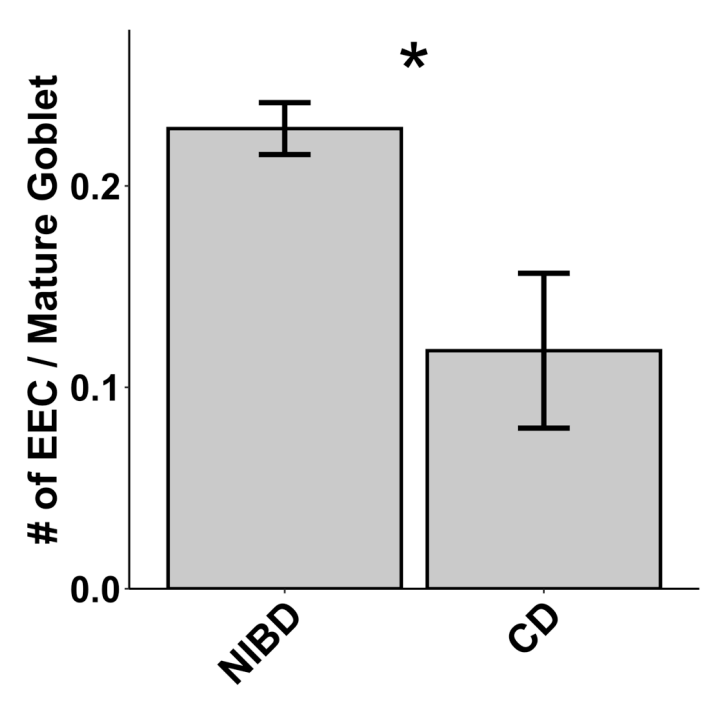

B

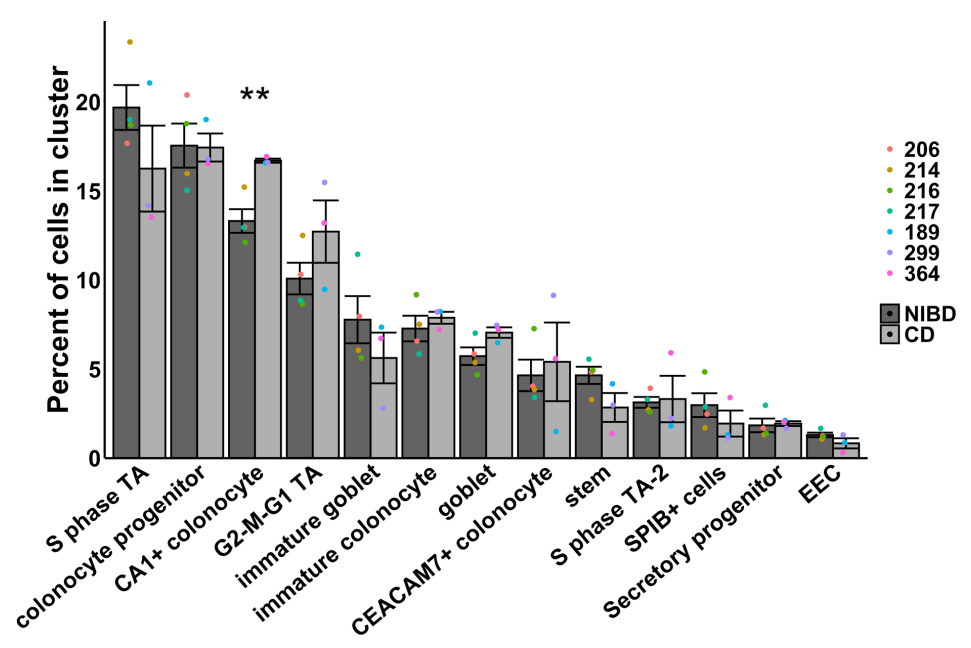

D

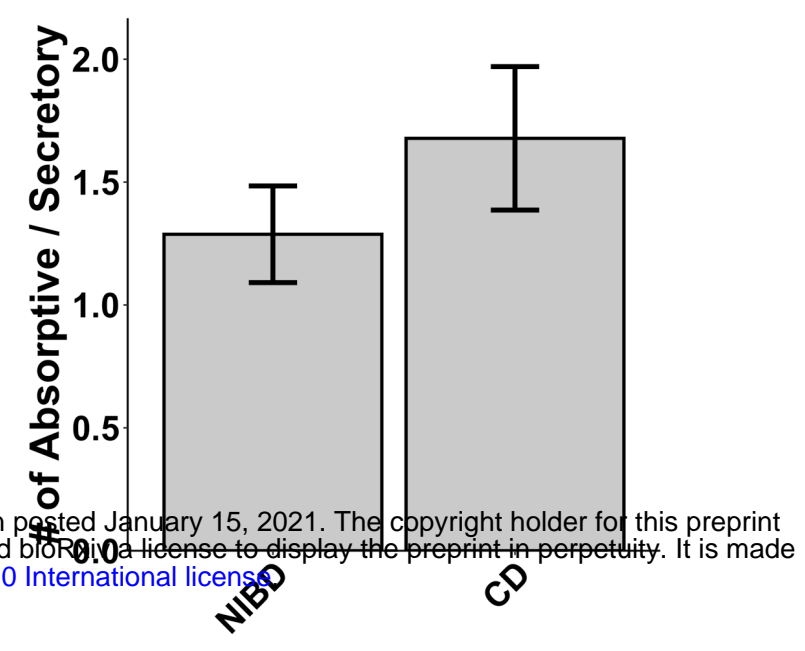

$\mathbf{F}$

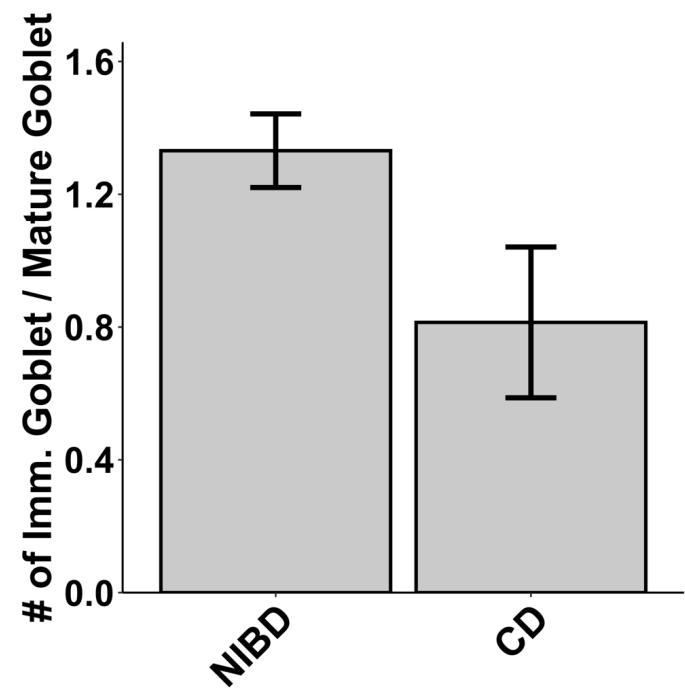




\section{Figure 3}

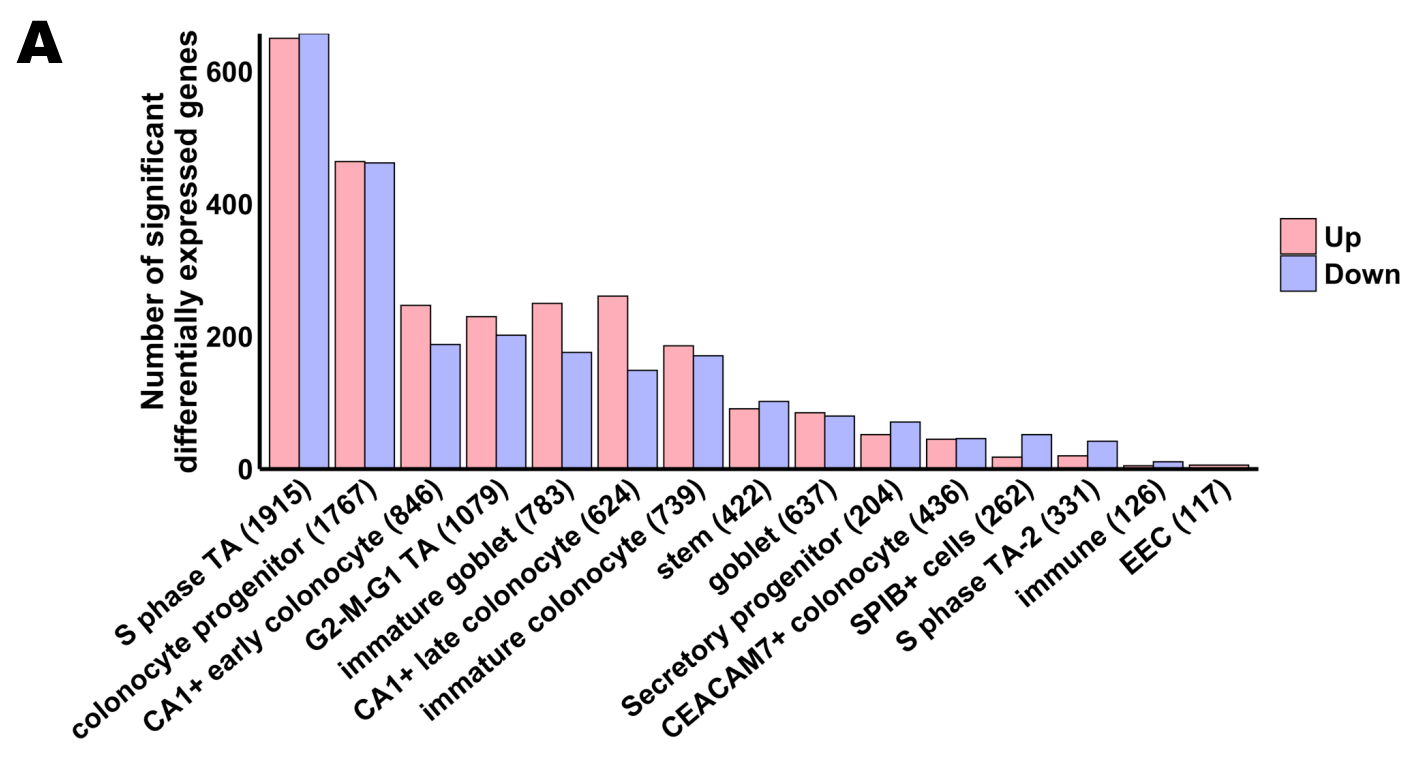

B CA1+ late colonocyte
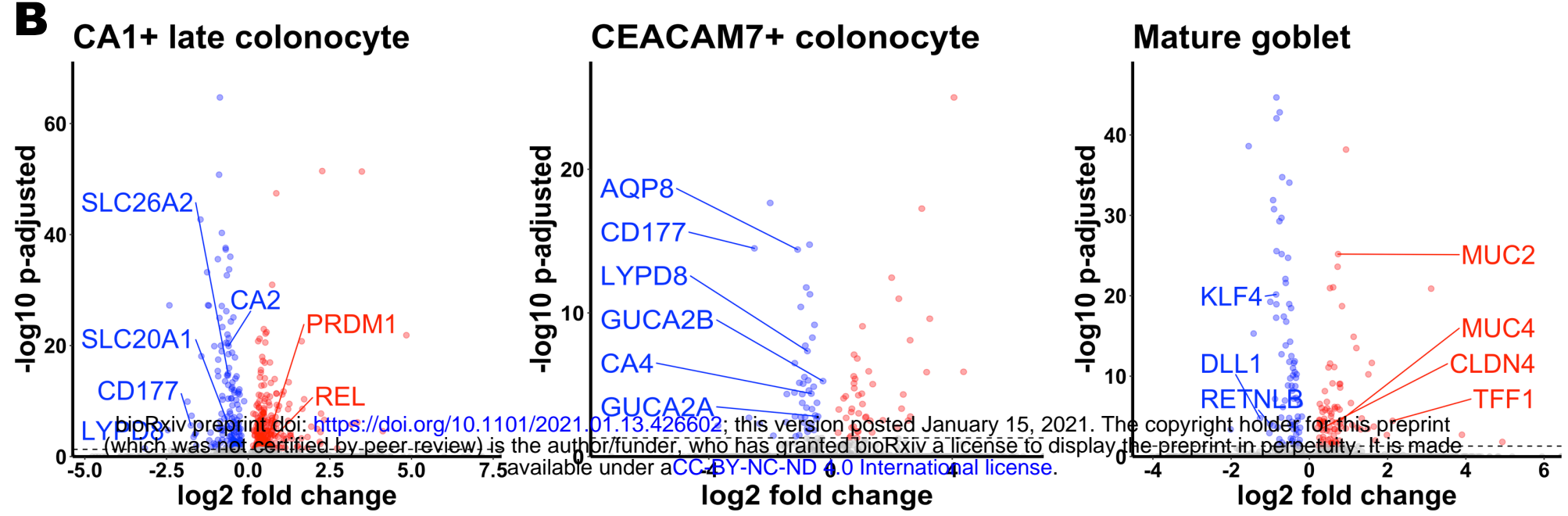

c

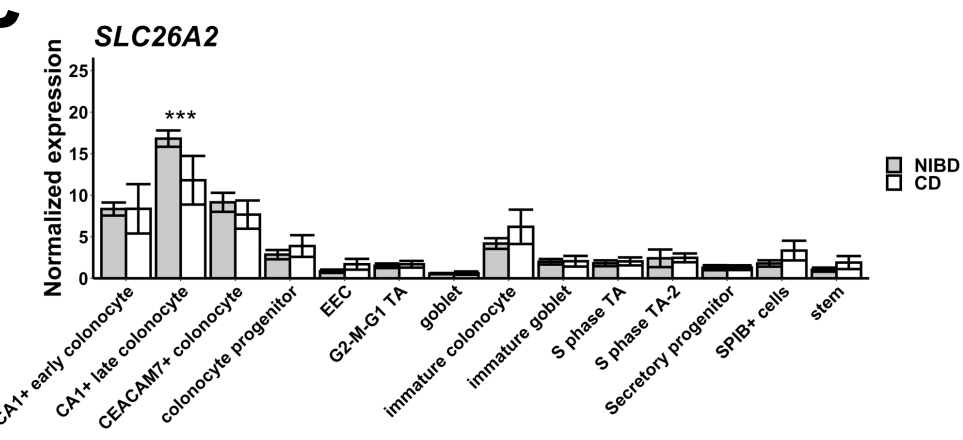

$\mathbf{E}_{\text {. } \quad C D 177}$

${ }_{C D}^{N 1 B D}$
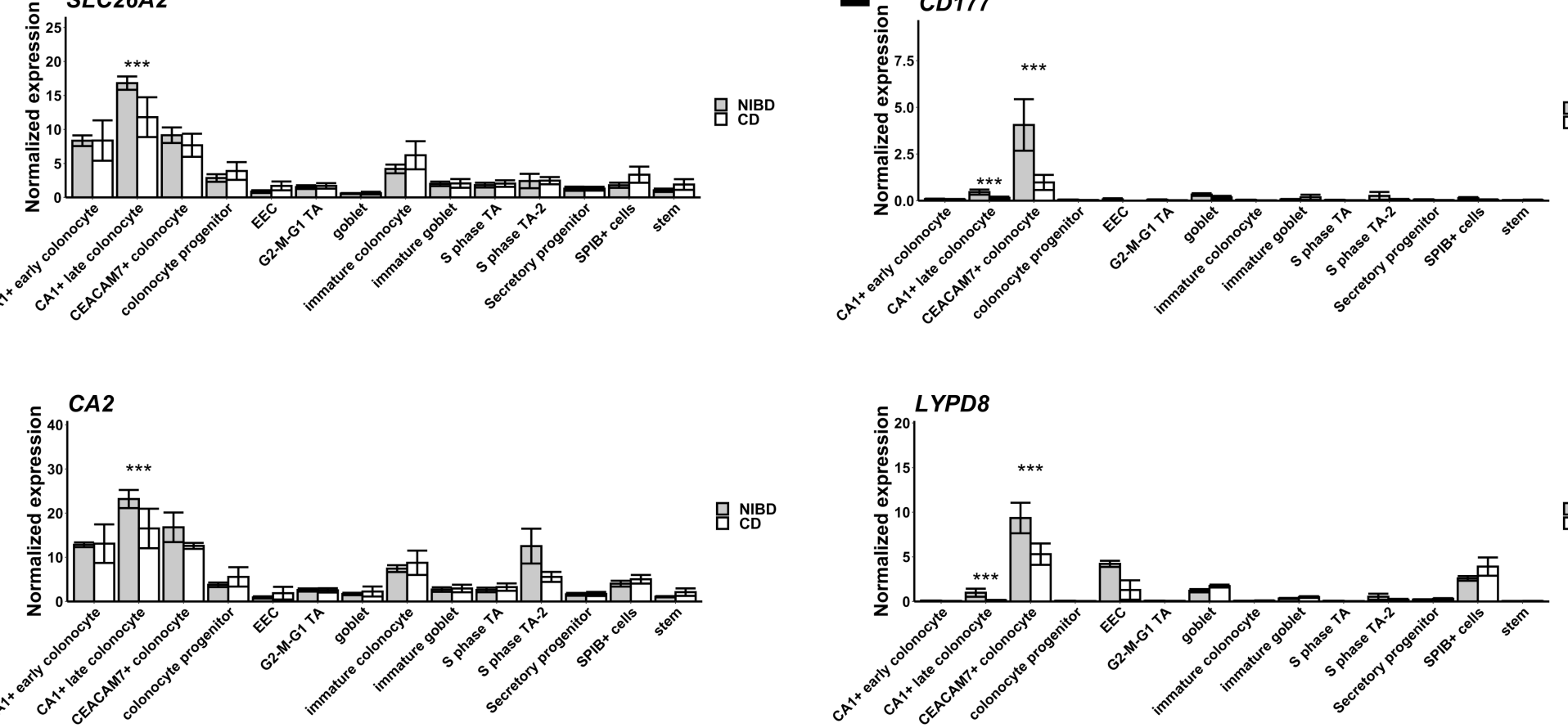

Q $\stackrel{\text { NIBD }}{C D}$

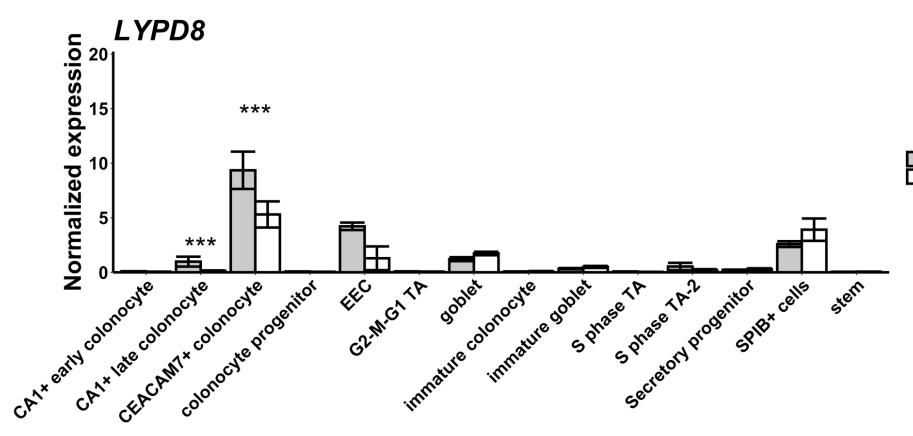

D
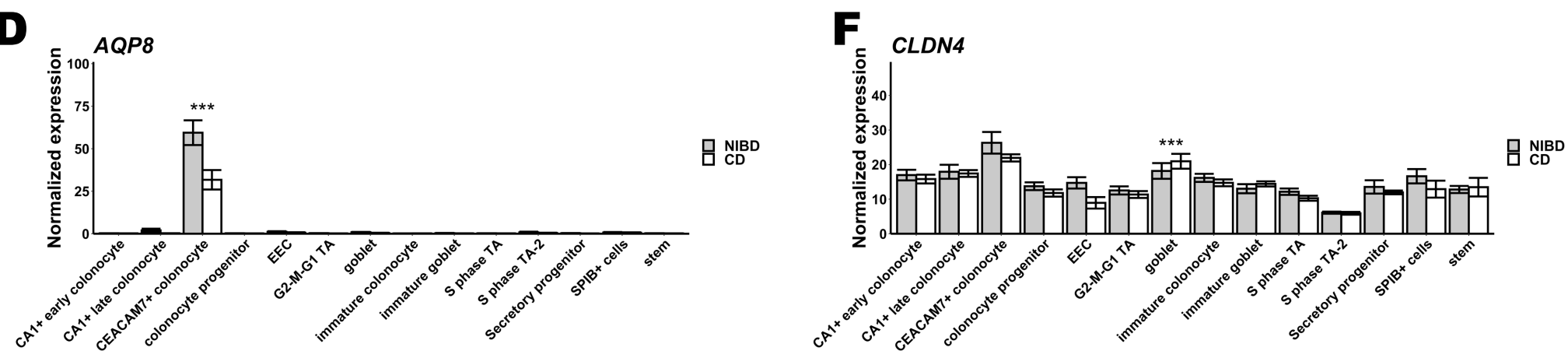


\section{Figure 4}

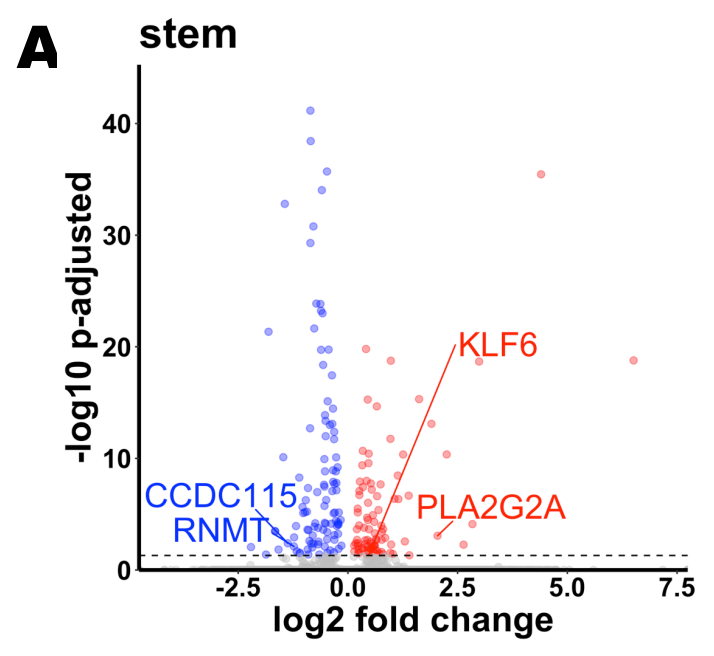

\section{B}

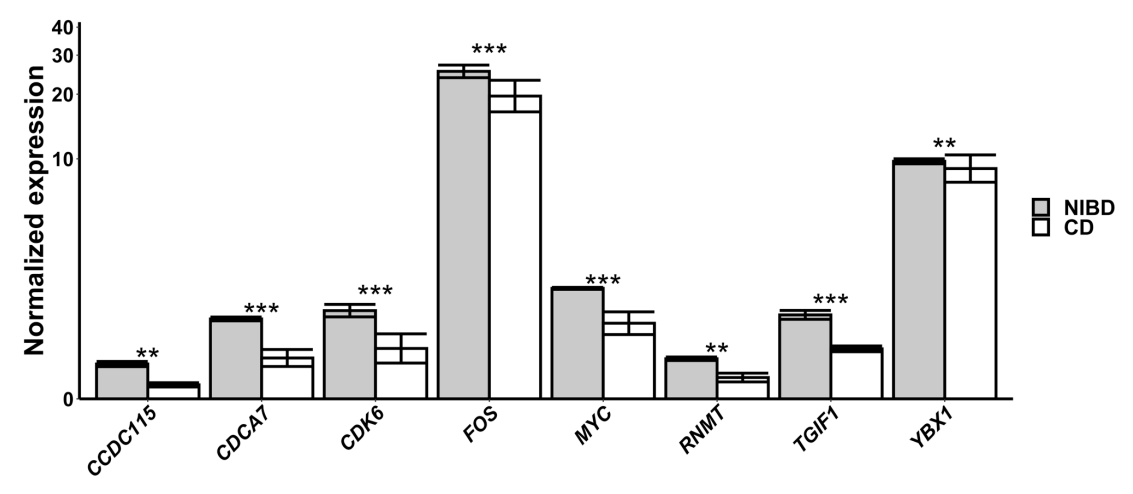

C
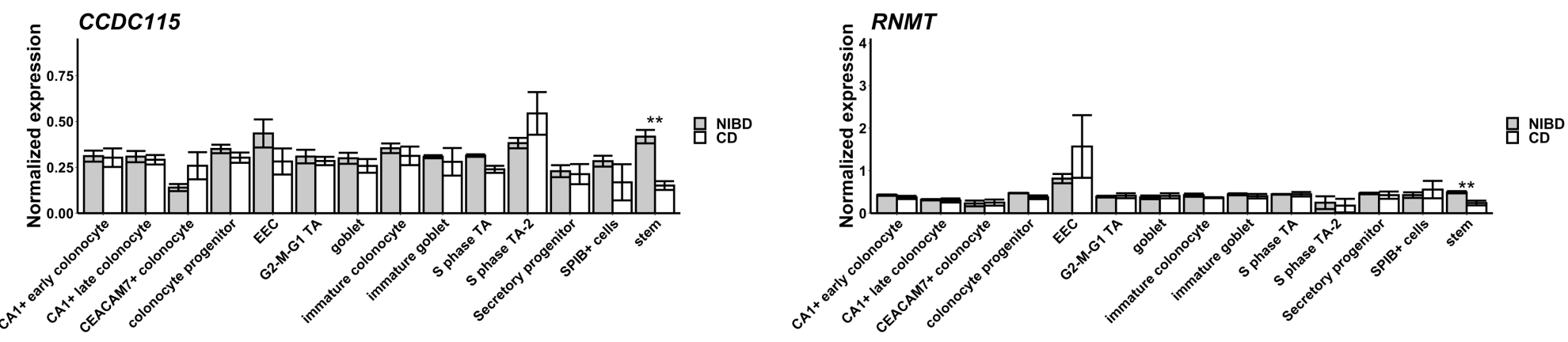

D

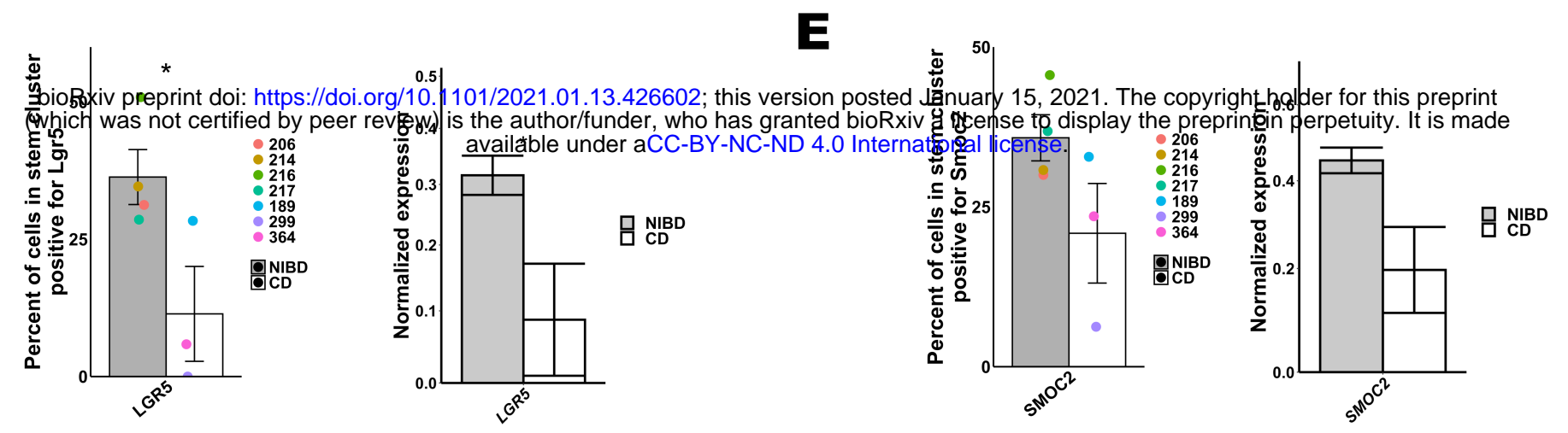

F
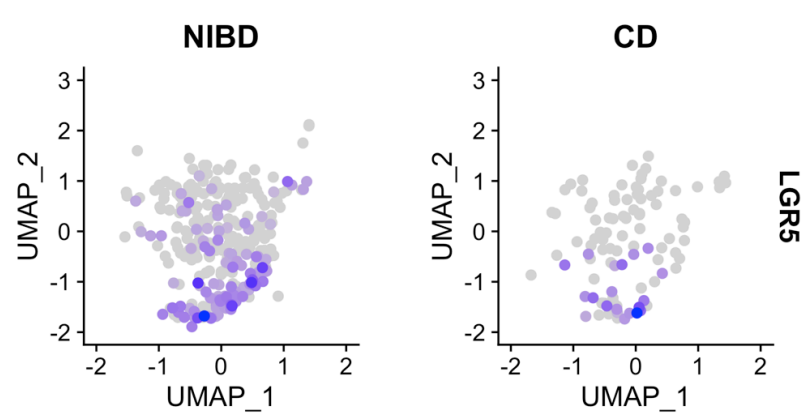

G
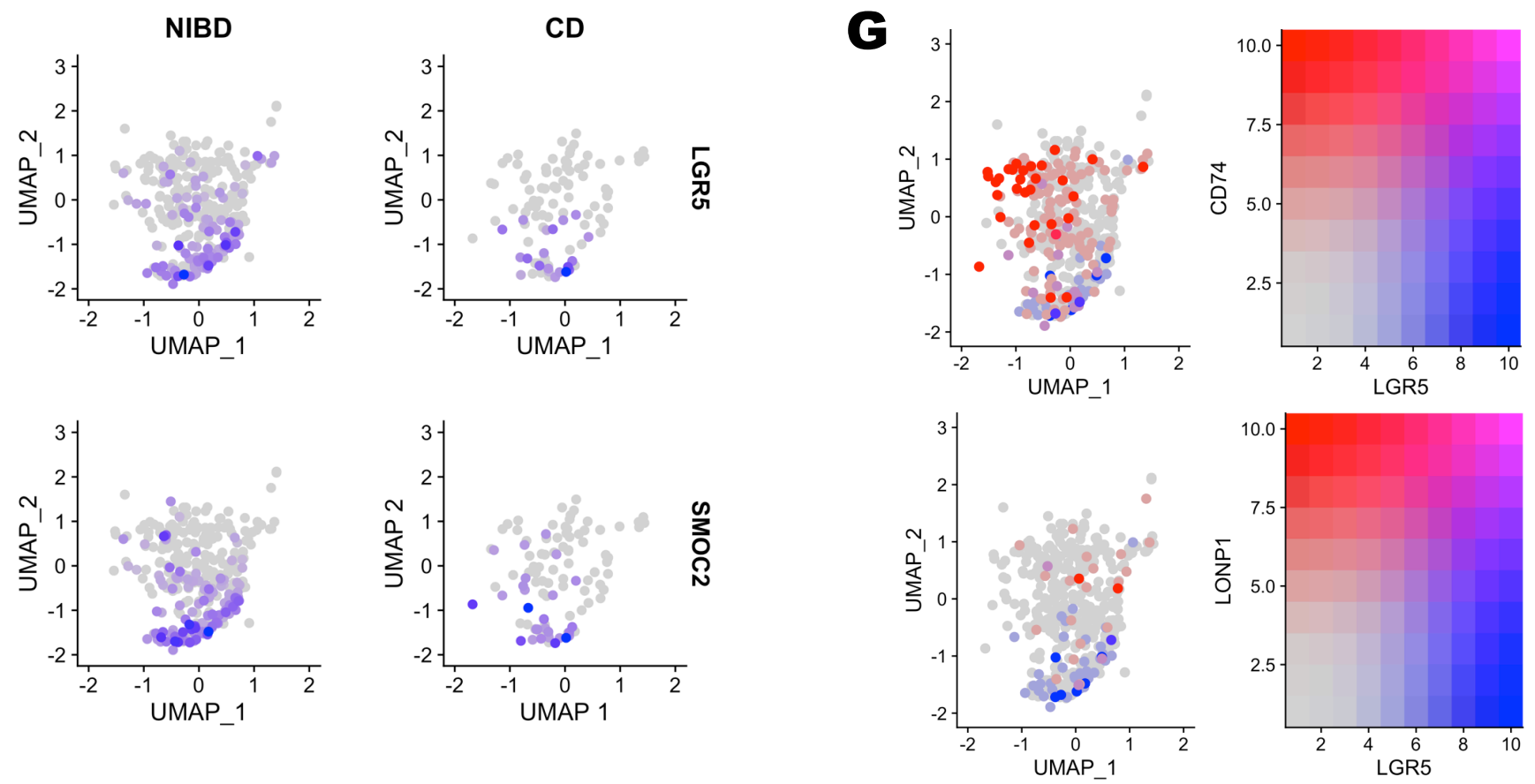

H
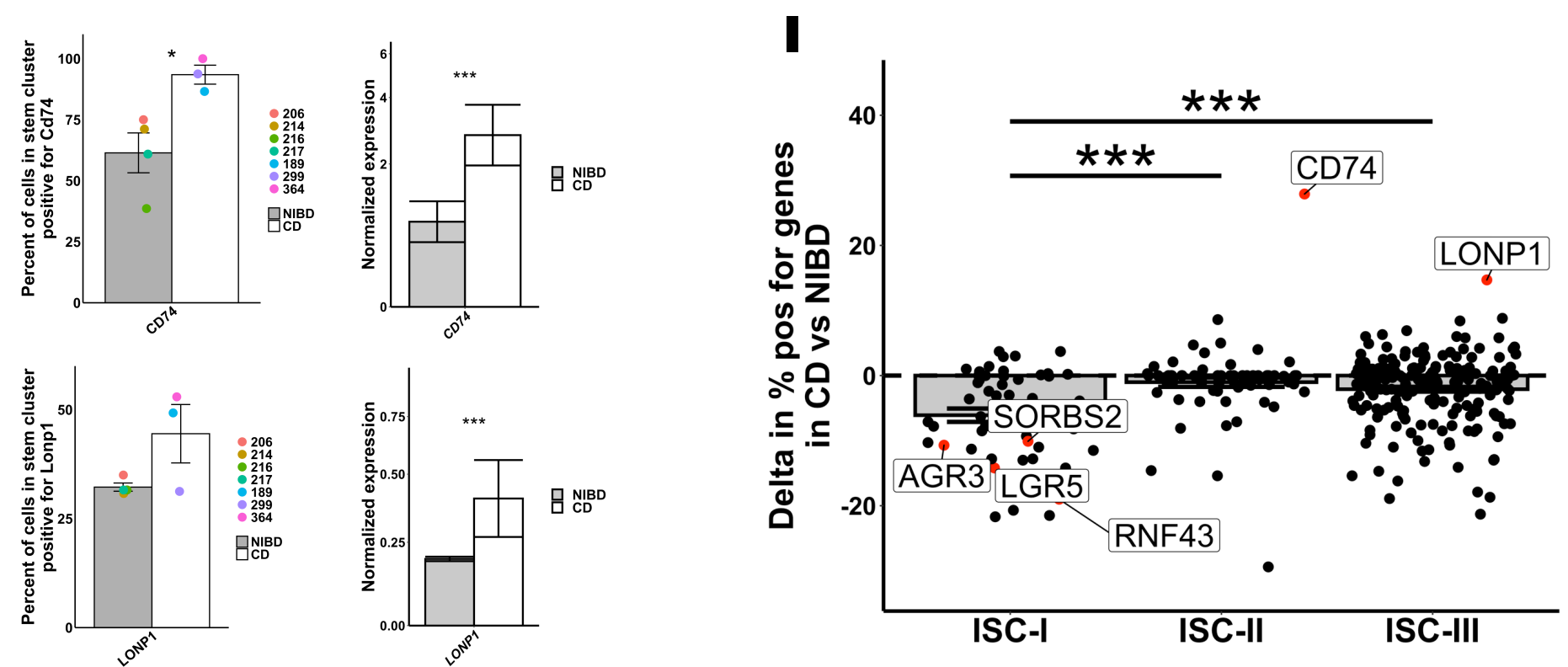
Figure 5
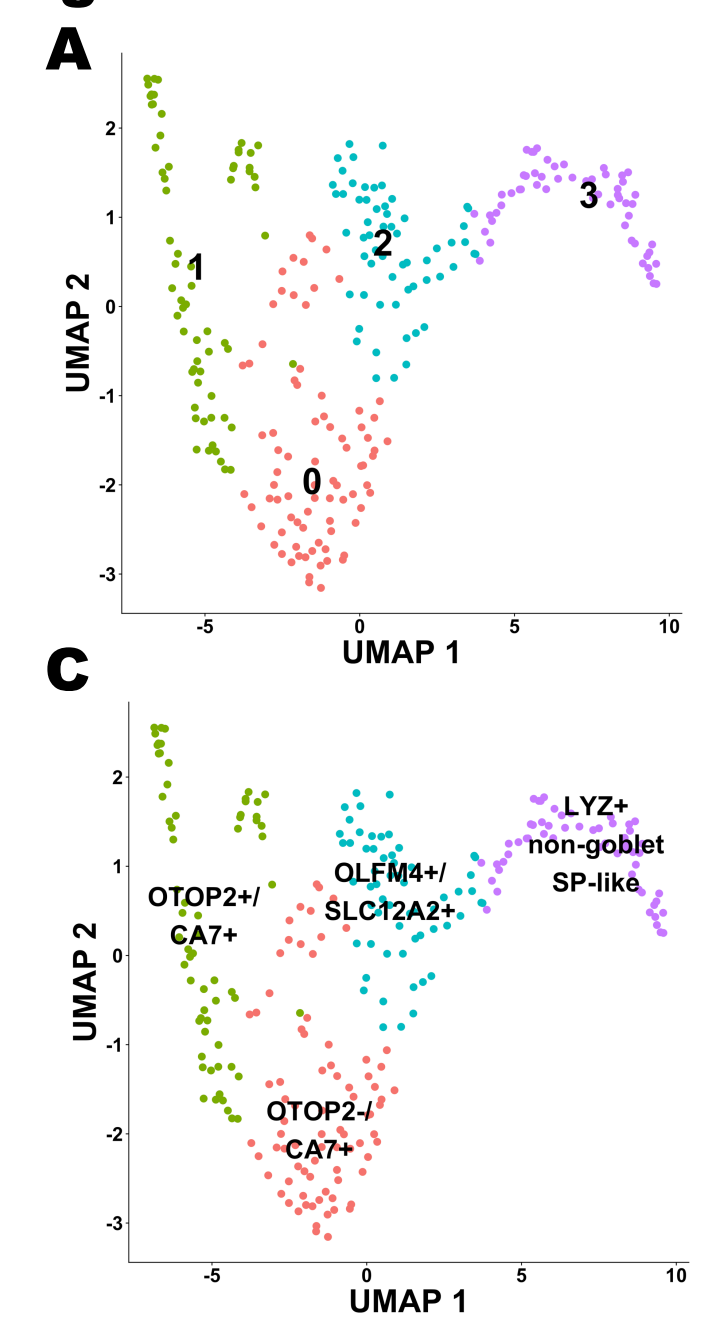

B
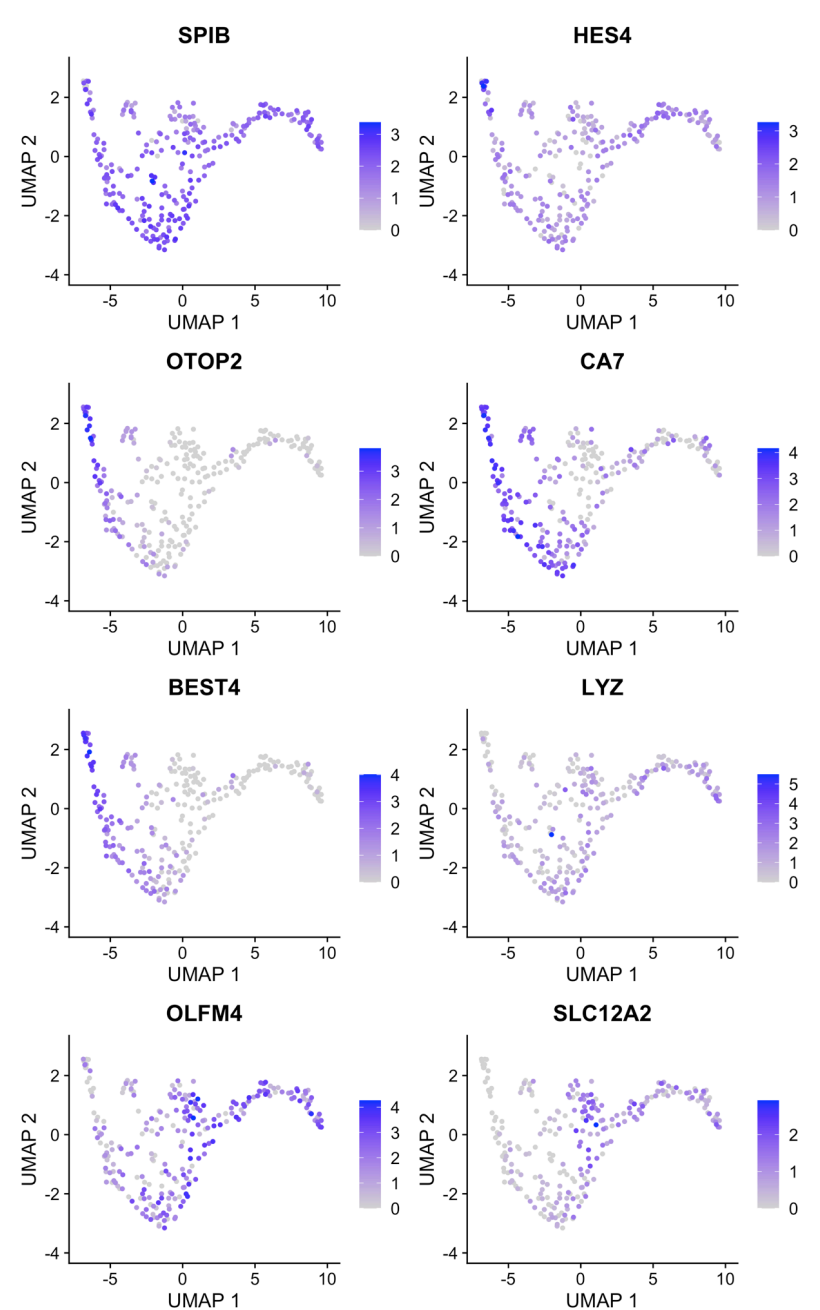

8 Top

3.48602; this version posted January 15, 2021.*The copyright holder for this preprint

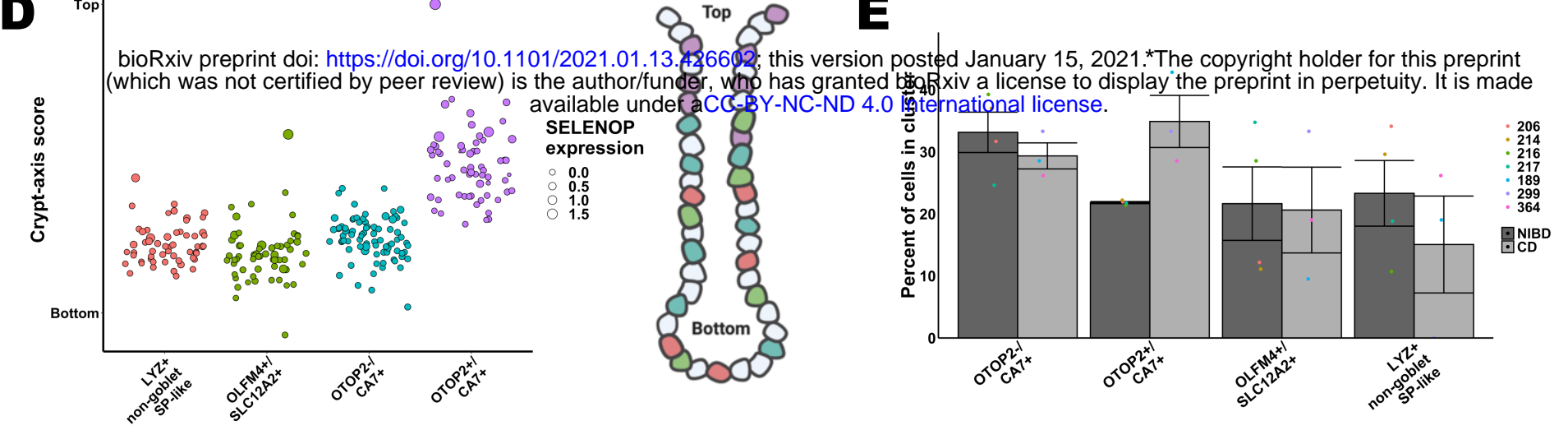

F

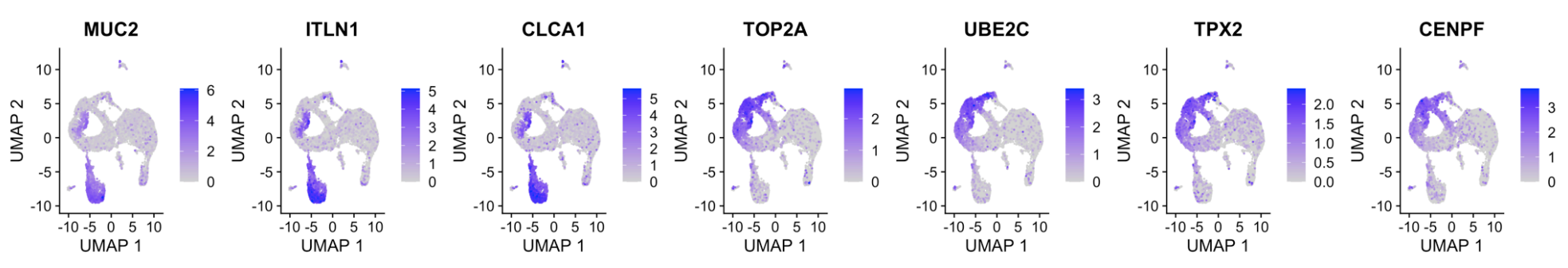

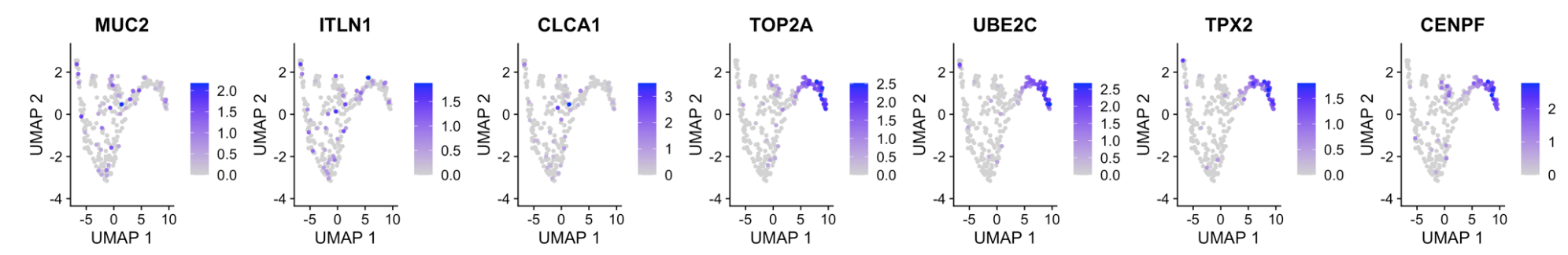

G

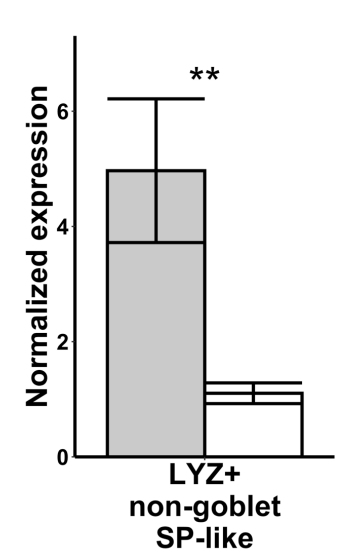

马 ${ }^{N D D}$
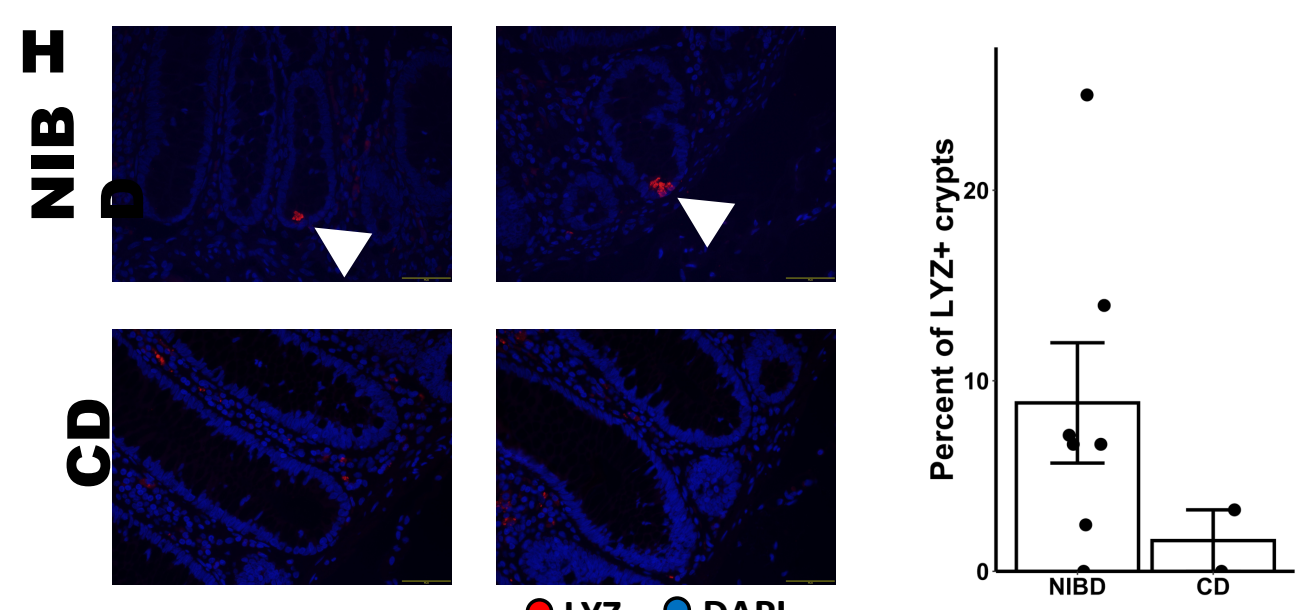

I

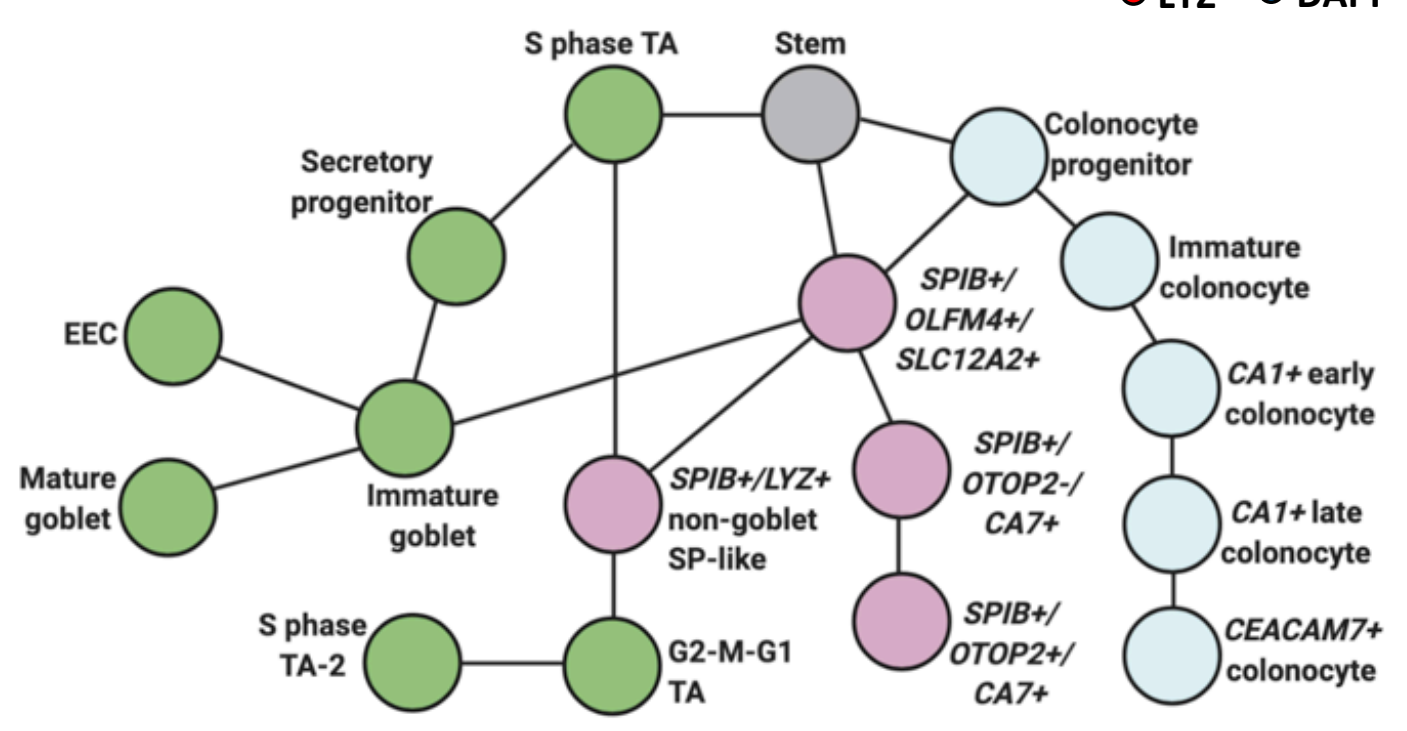


Figure 6

$\mathbf{A}$

stem
S phase TA
G2-M-G1 TA
S phase TA-2
Secretory progenitor
immature goblet
goblet
EEC
colonocyte progenitor
immature colonocyte
CA1+ early colonocyte
CA1+ late colonocyte
CEACAM7+ colonocyte
SPIB+ cells

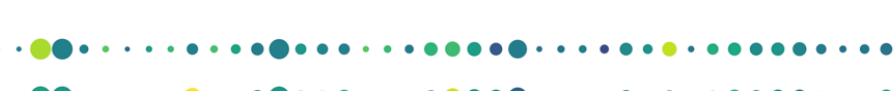

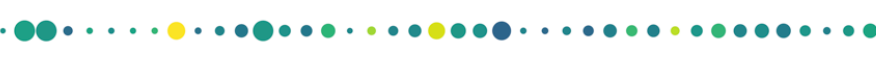

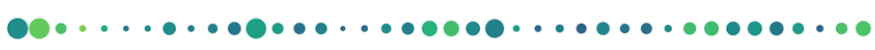

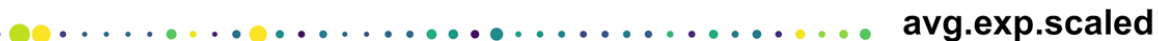

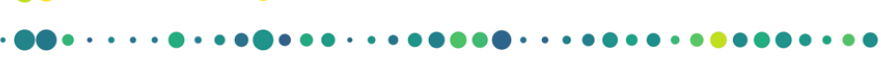

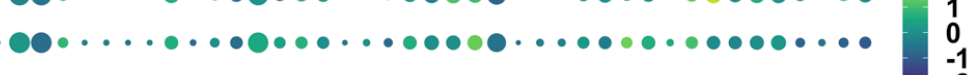

$\bullet$............................ ${ }_{-2}^{-1}$



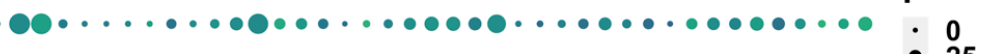

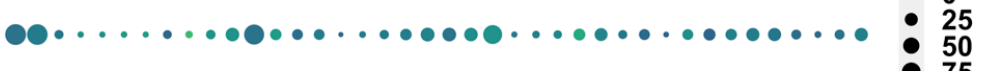

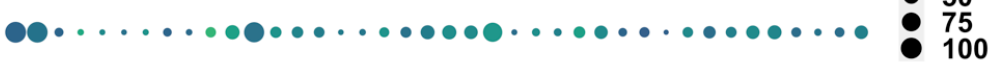

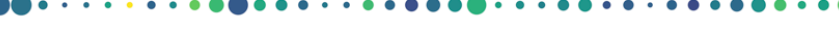

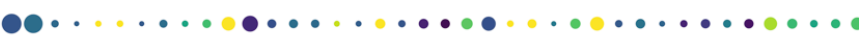

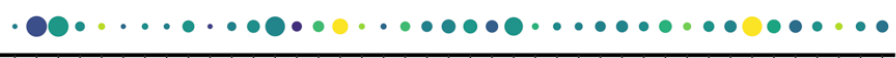

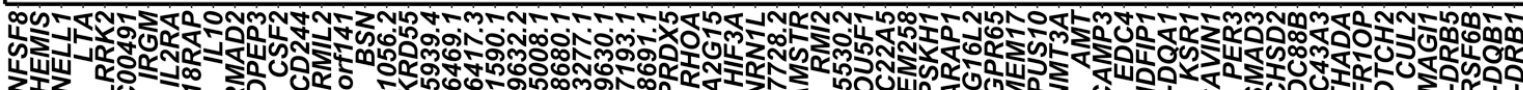

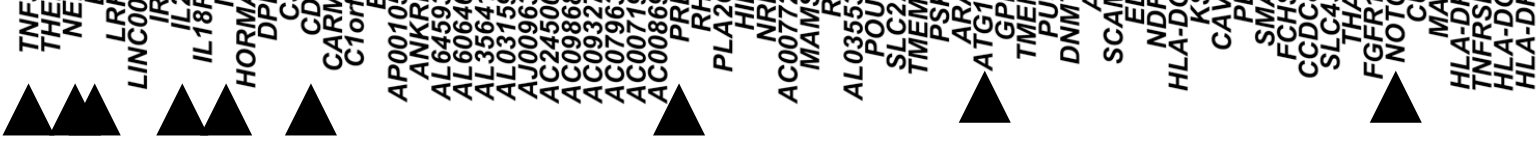

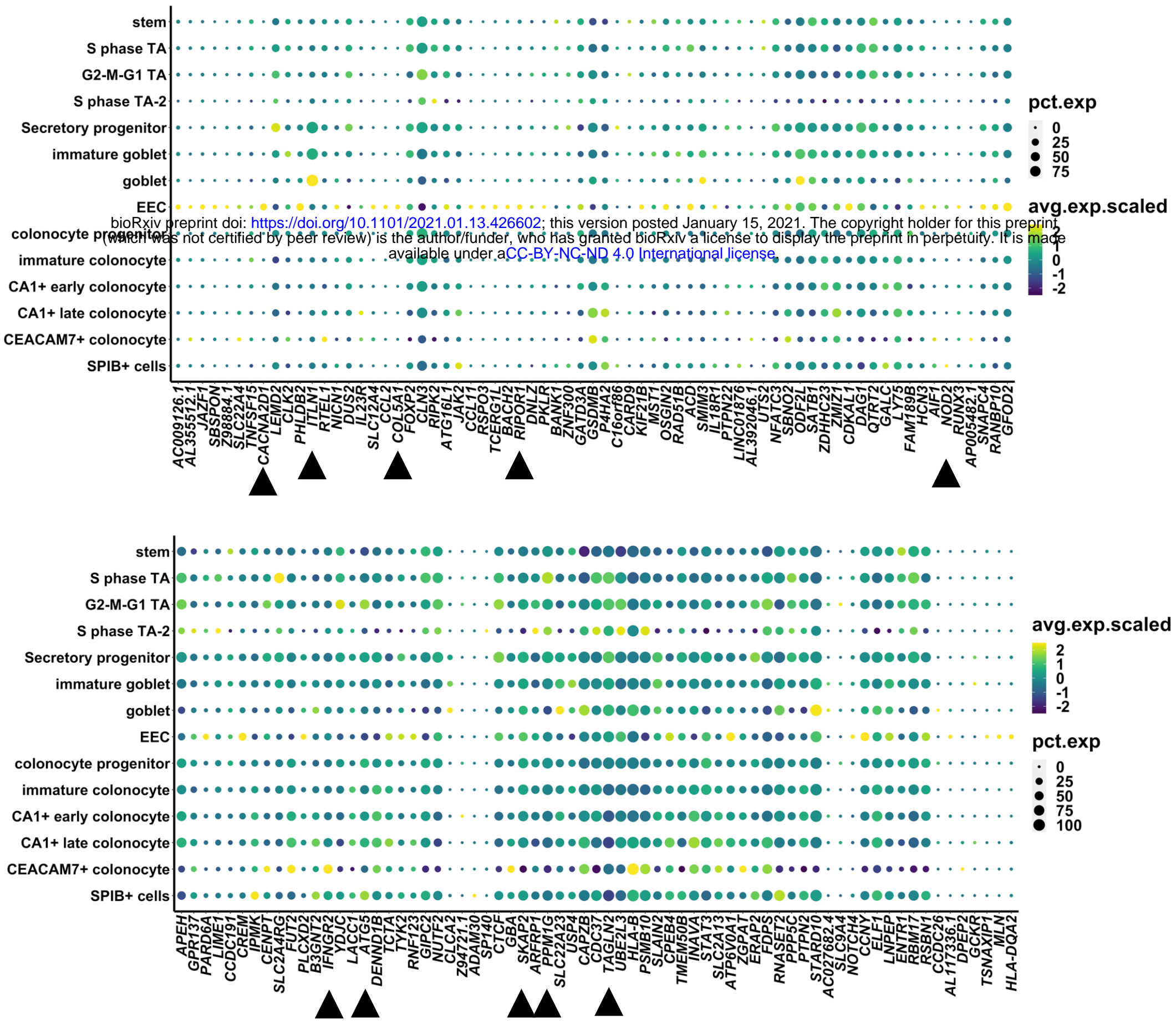

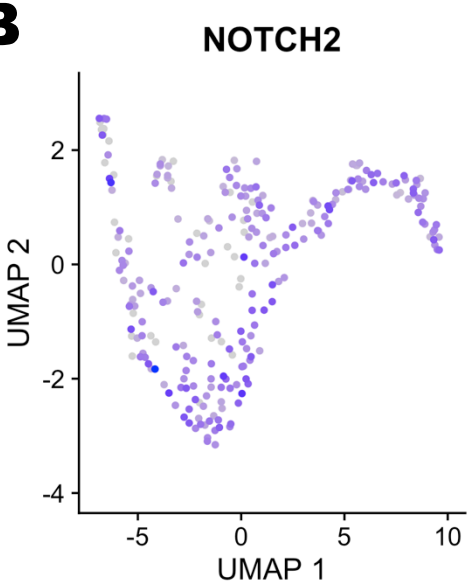

ATG16L2

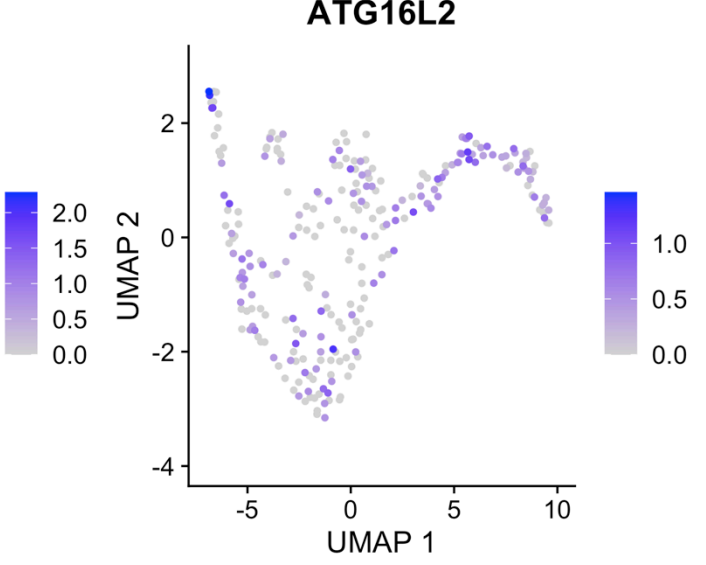




\section{Figure 7}

$\mathbf{A}$

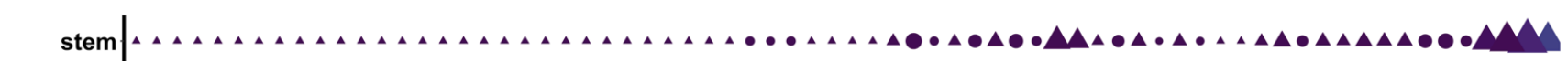

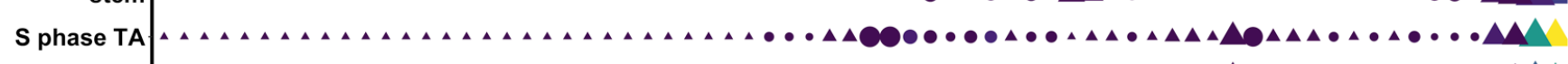

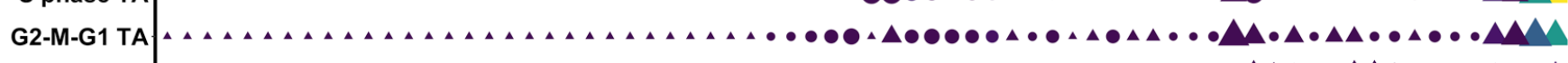

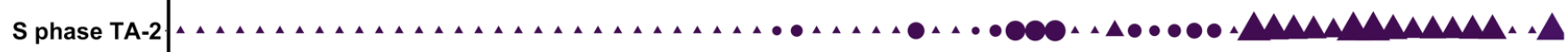

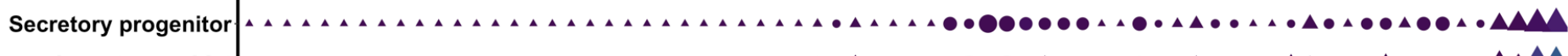

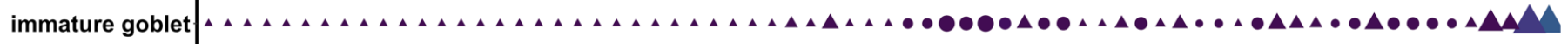

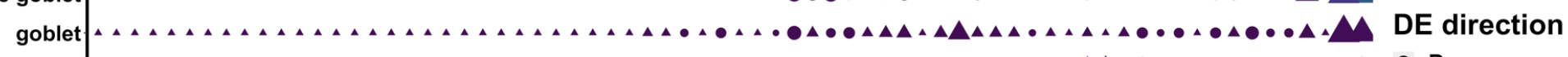

colonocyte progenitor

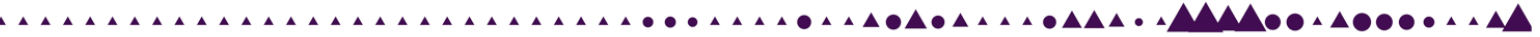

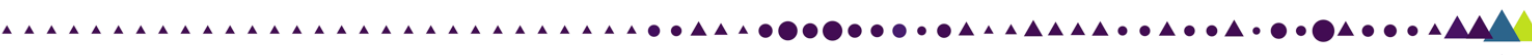

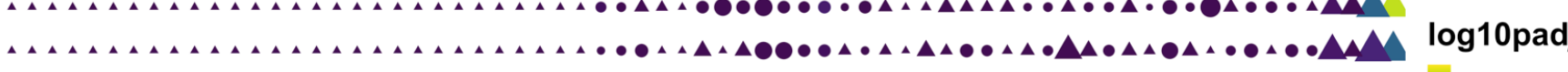

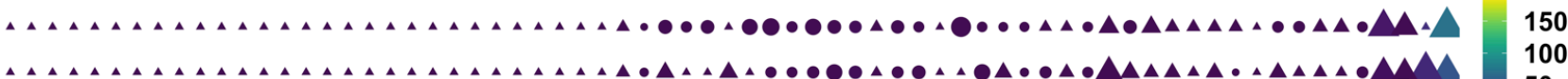

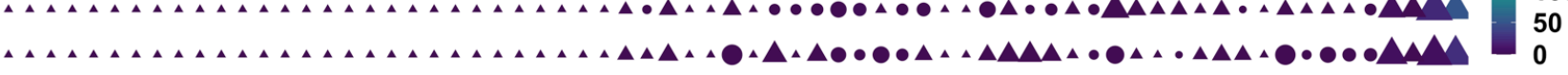

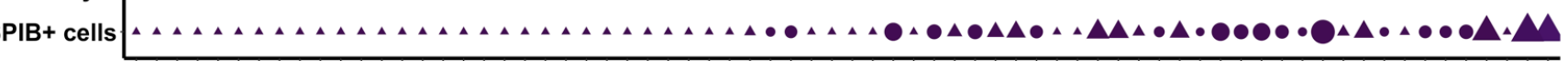

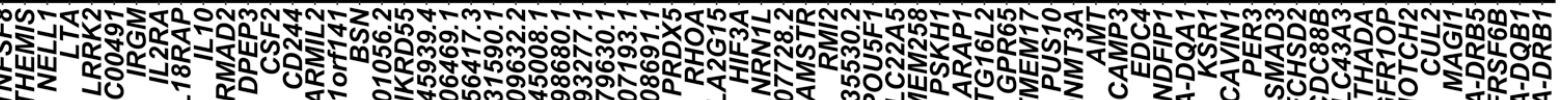

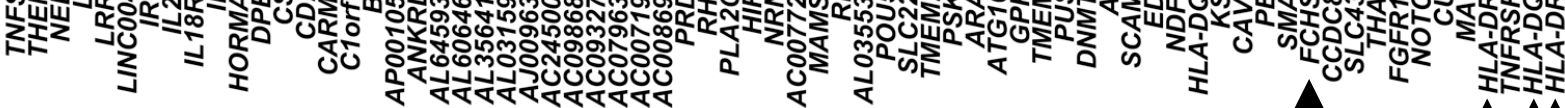

\section{$\Delta \stackrel{\Lambda}{\wedge}$}

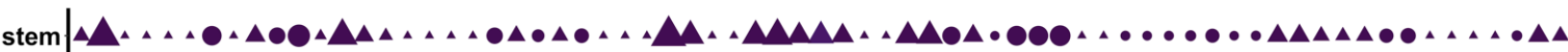

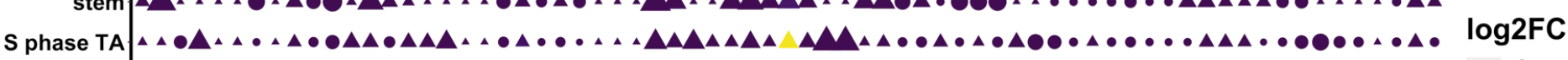

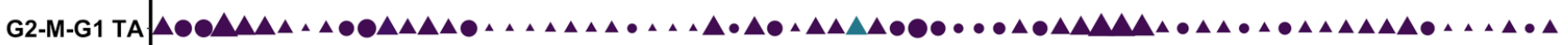

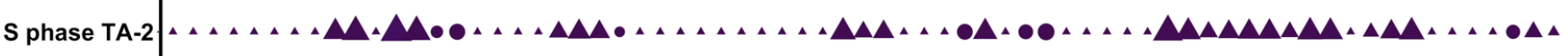

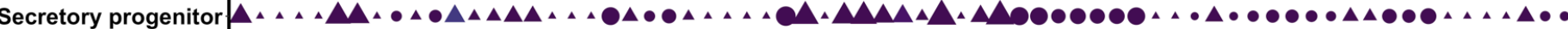

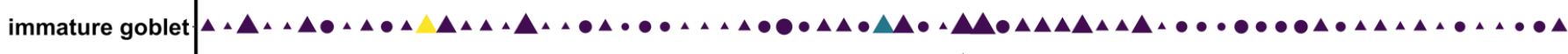

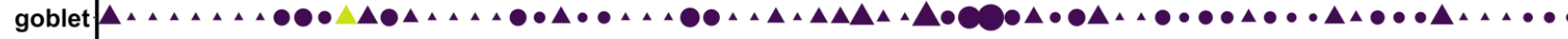

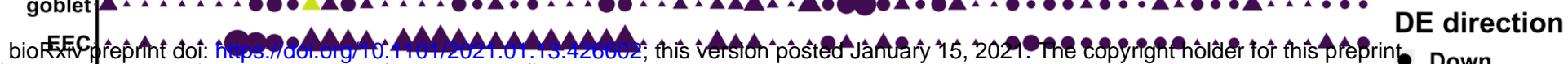

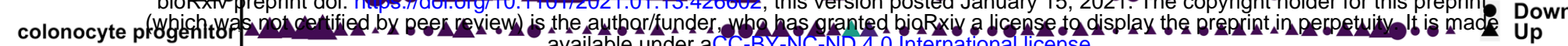

immature colonocyte

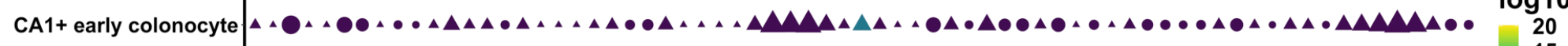

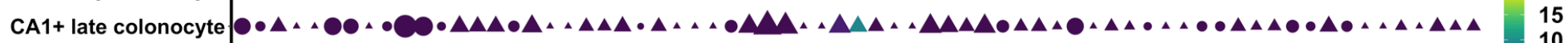

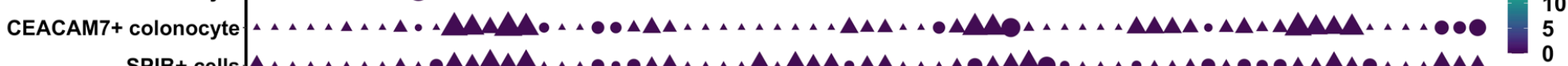

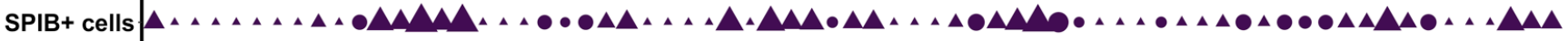

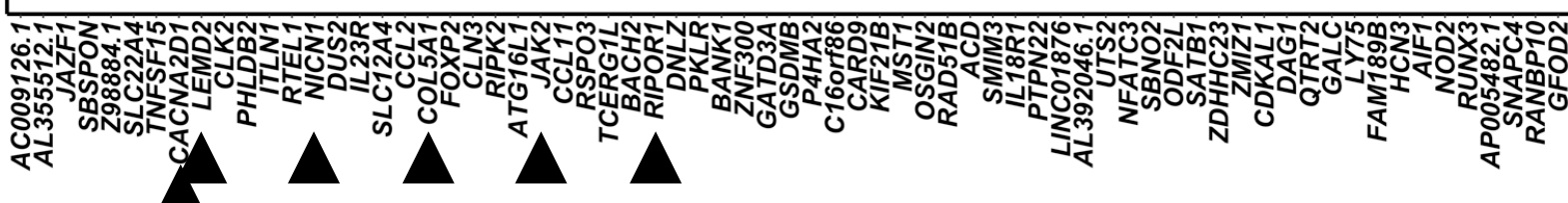

4

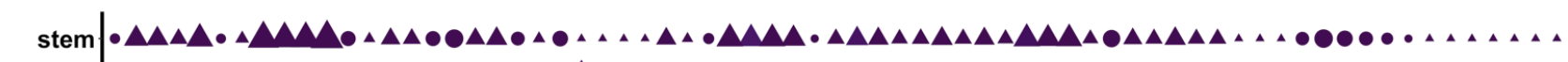

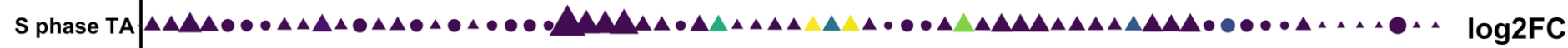

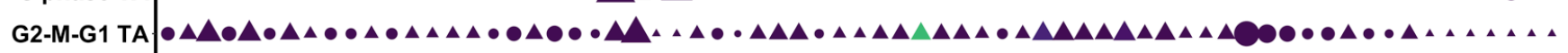

S phase TA-2 $\triangle \bullet \circ \Delta M$

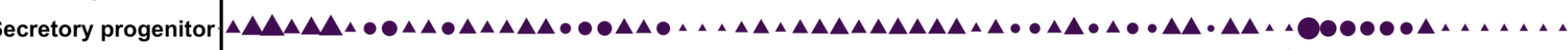

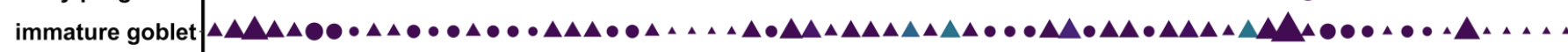
EEC $\triangle \bullet$ СООООО

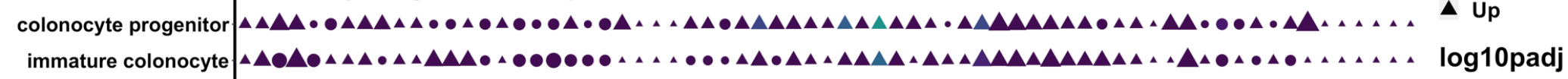

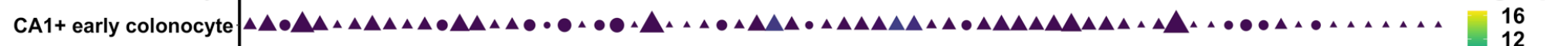

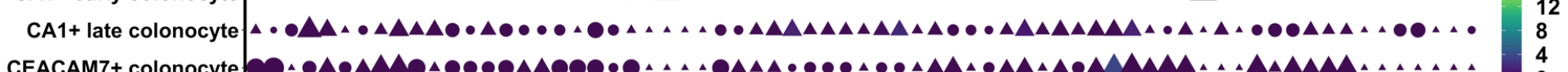

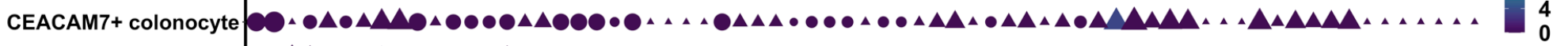

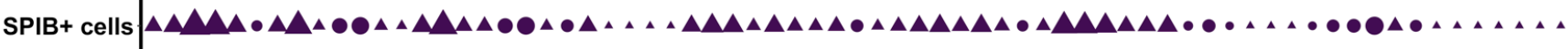

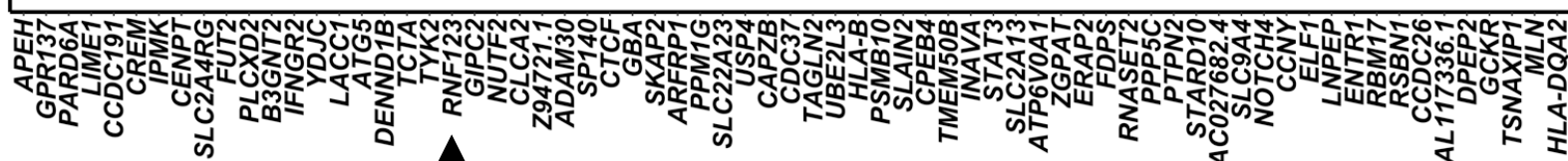

B

Key findings in CD

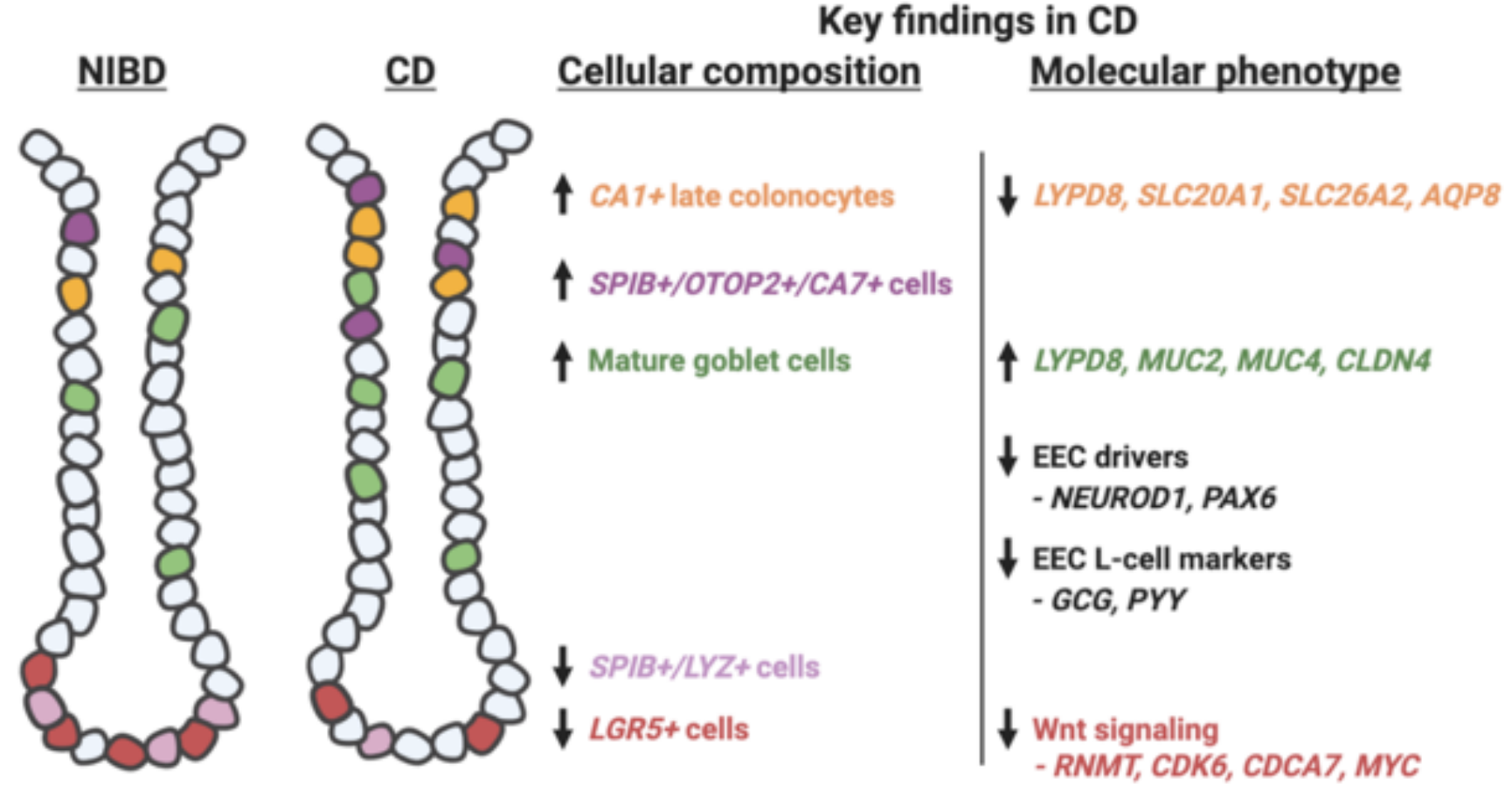




\section{Supplemental Figure 1}

A

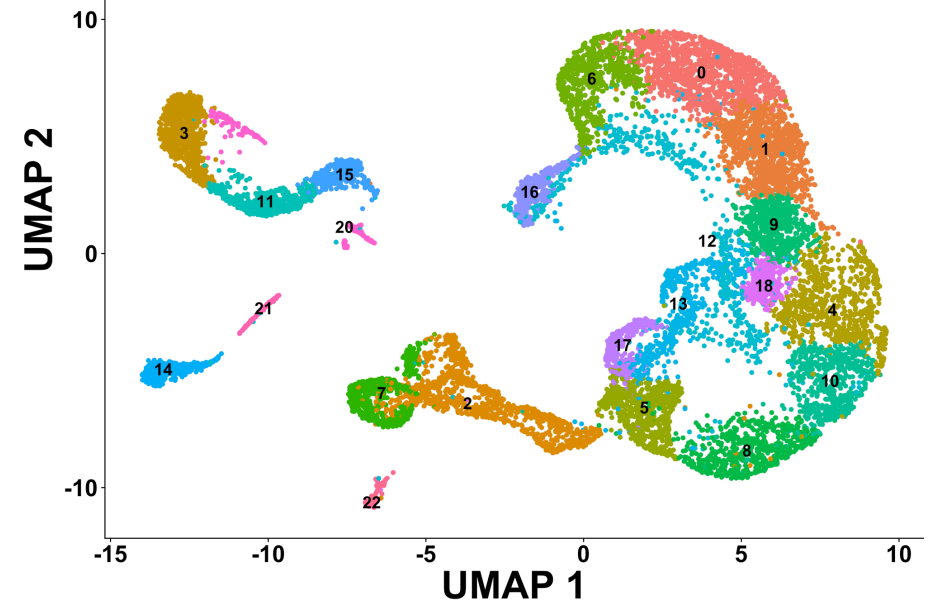

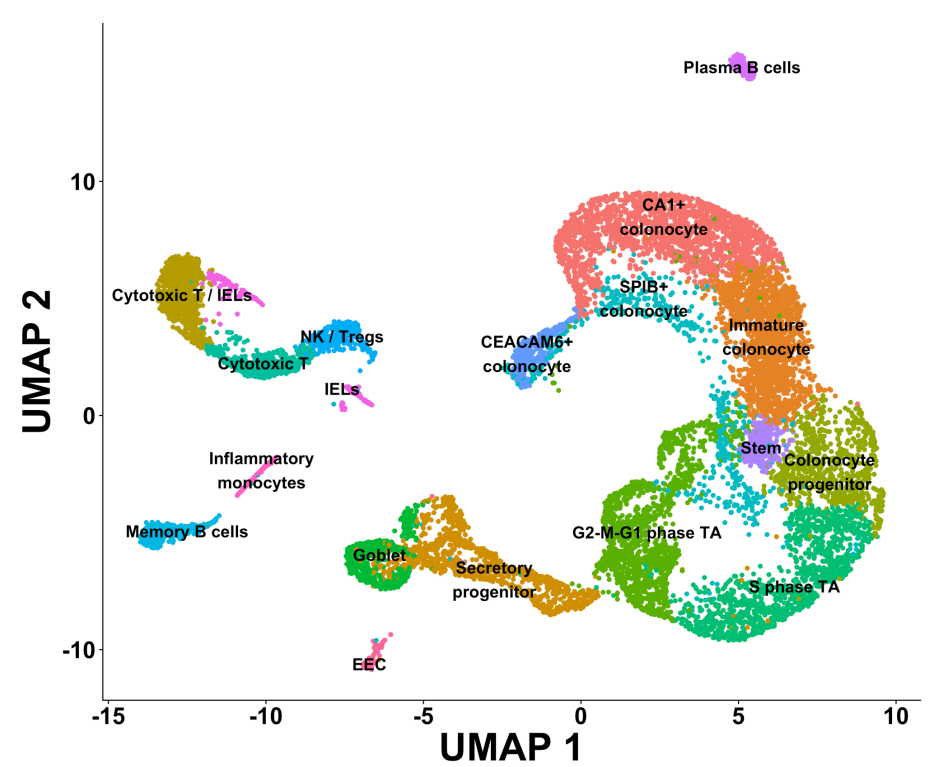

B

Pan-T

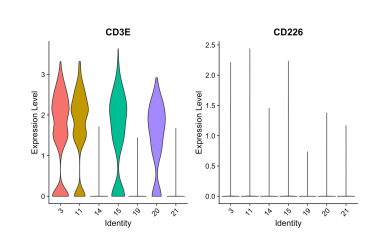

IELS
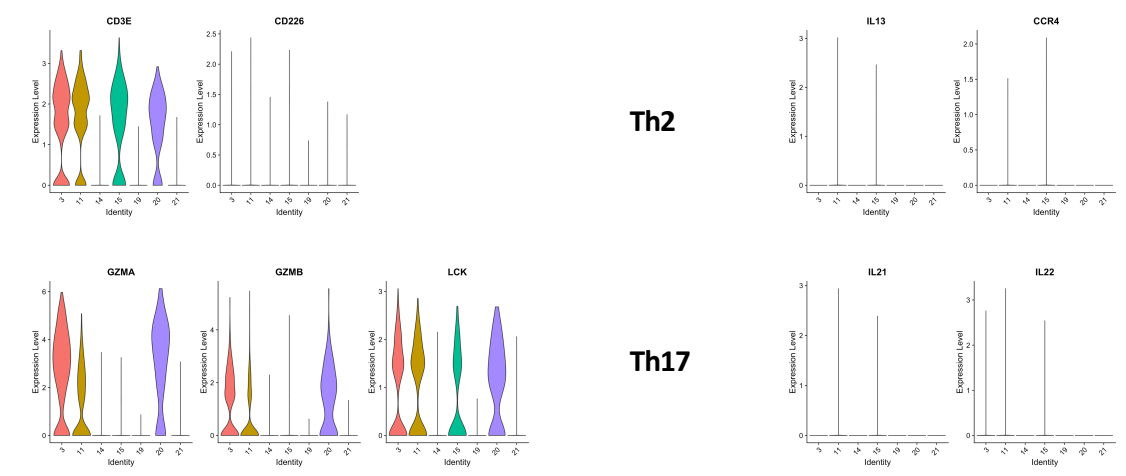

Pan-B

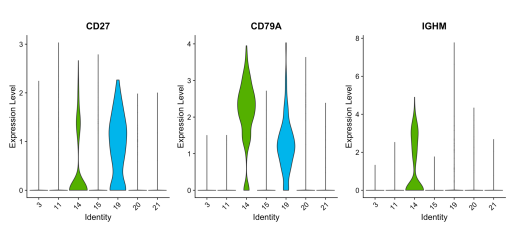
(which was not certified by peer review) is the author/funder, who has granted bioRxiv a license to display the preprint imaperpetuity. Itis mad
Treg

Cytotoxic

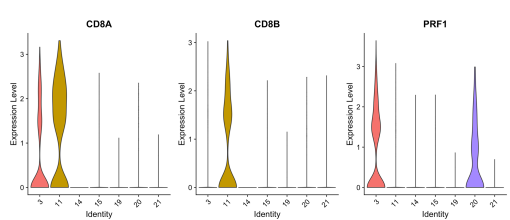

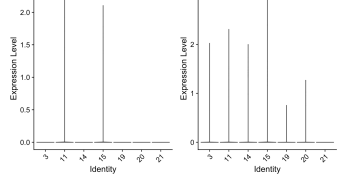

Th17

Th1

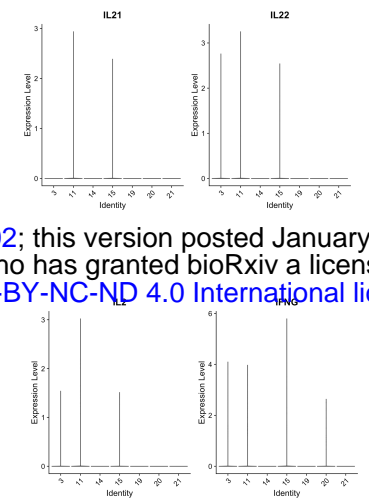

NK
Plasma B

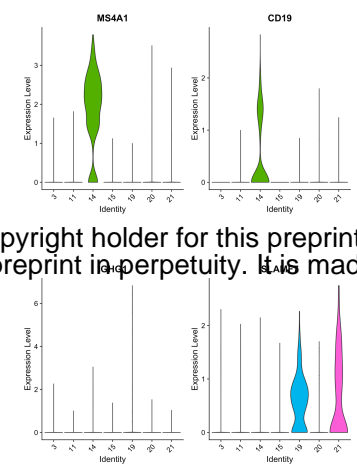

Inflammatory Monocytes
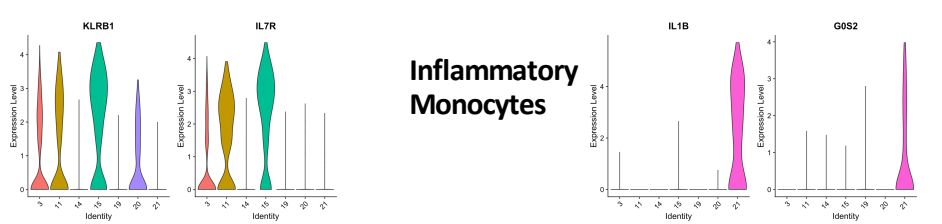


\section{Supplemental Figure 2}

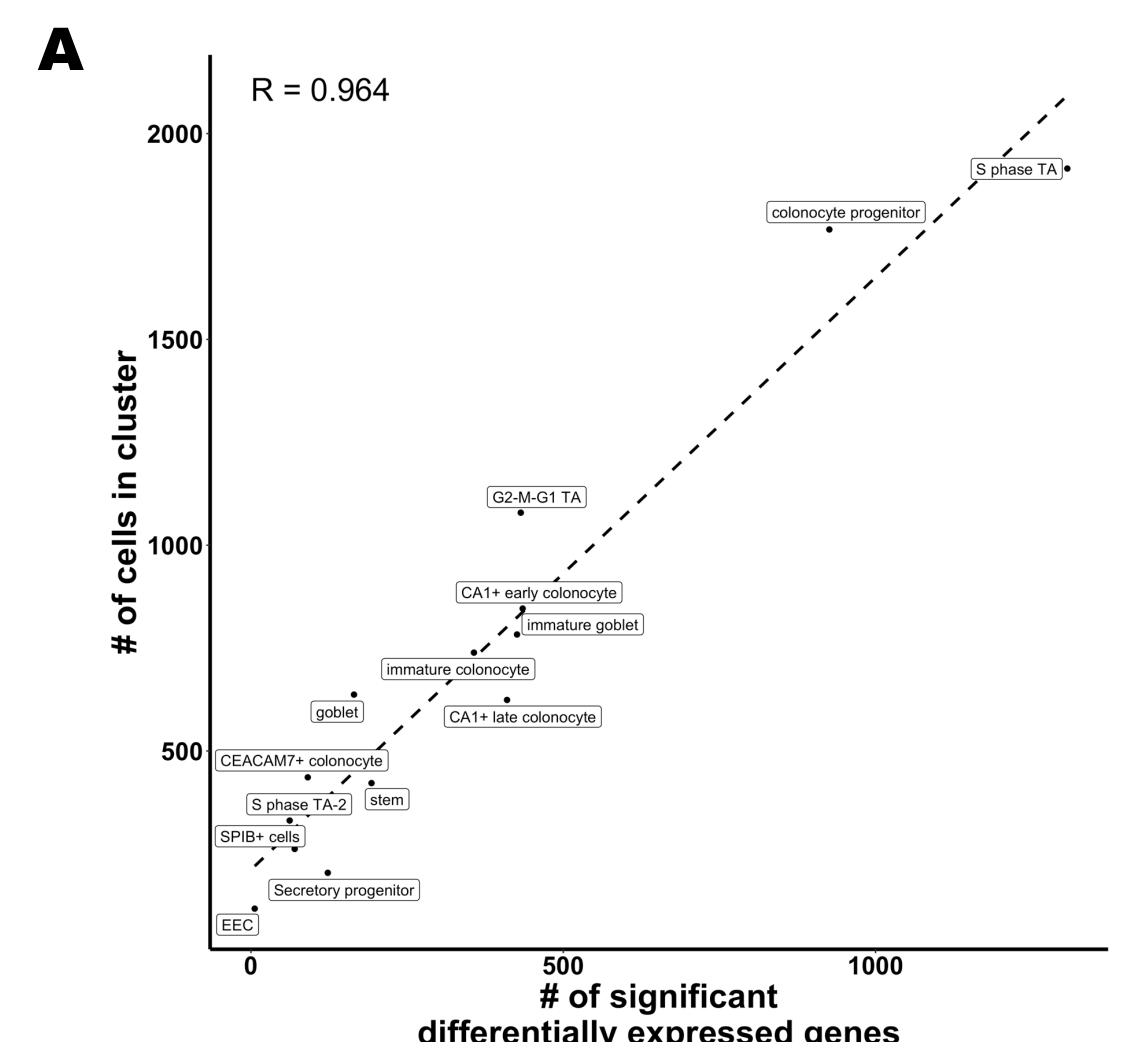

differentially expressed genes

bioRxiv preprint doi: https://doi.org/10.1101/2021.01.13.426602; this version posted January 15, 2021. The copyright holder for this preprint (which was not certified by peer review) is the author/funder, who has granted bioRxiv a license to display the preprint in perpetuity. It is made
available under aCC-BY-NC-ND 4.0 International license. 
Supplemental Figure 3

A
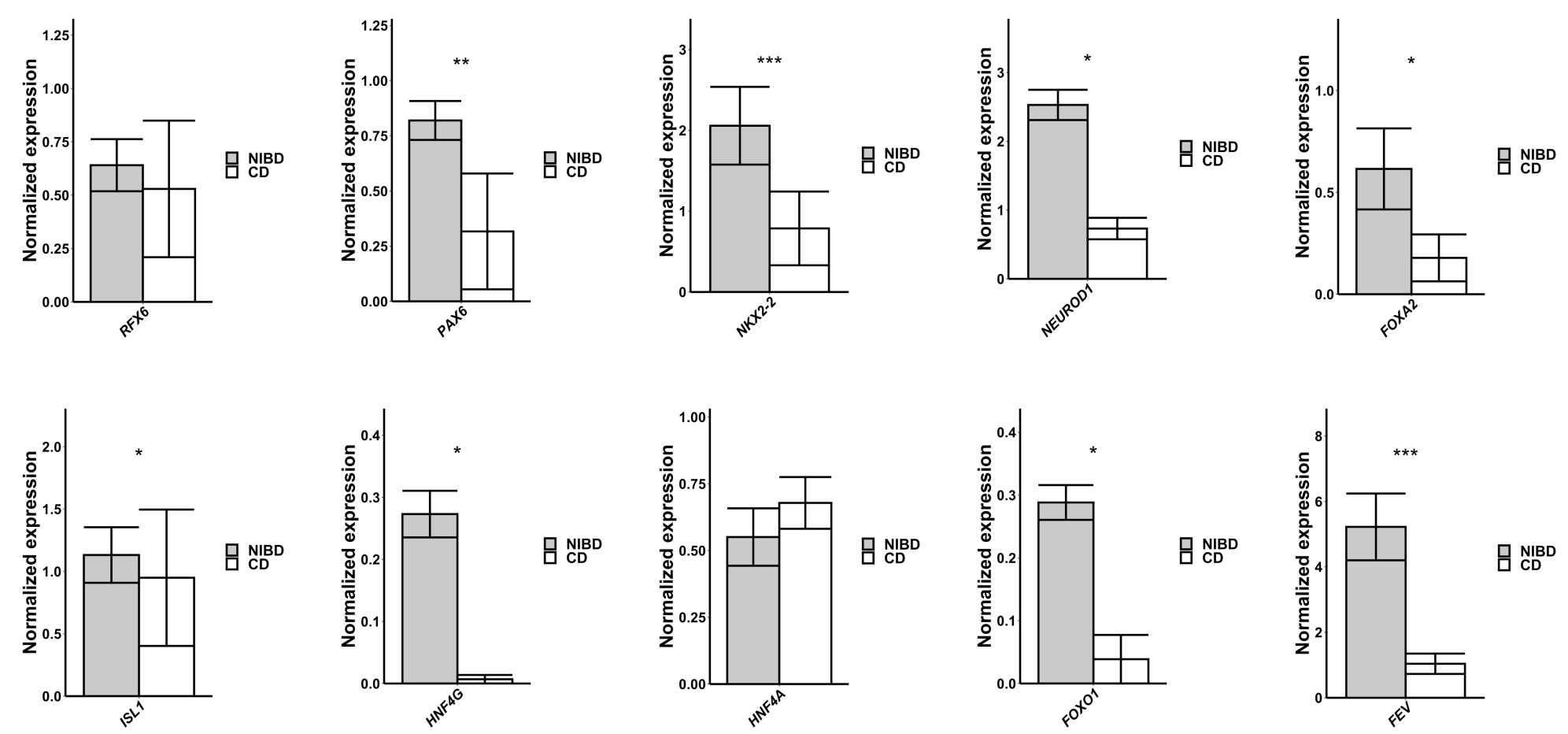

B
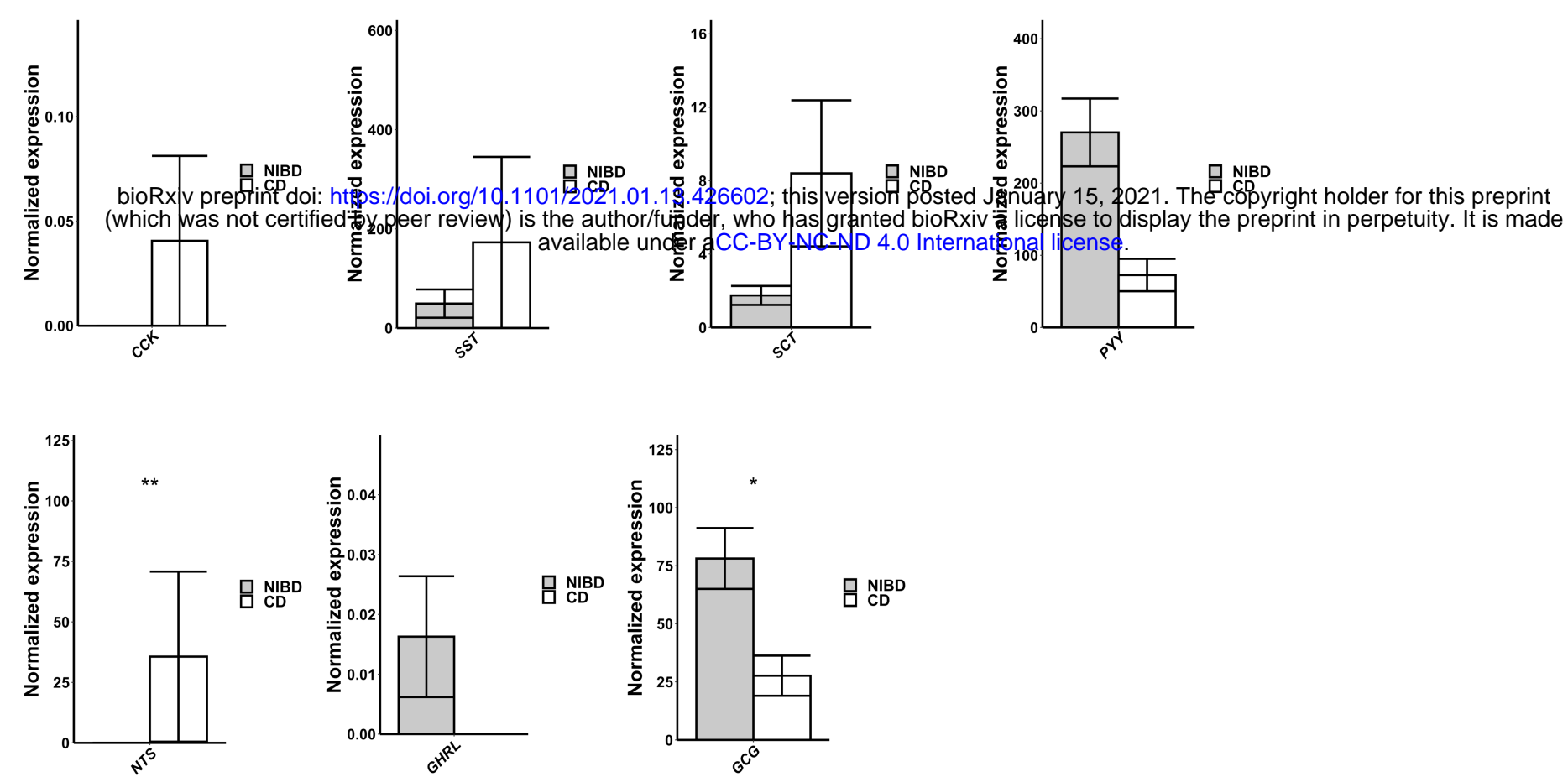
Supplemental Figure 4

A
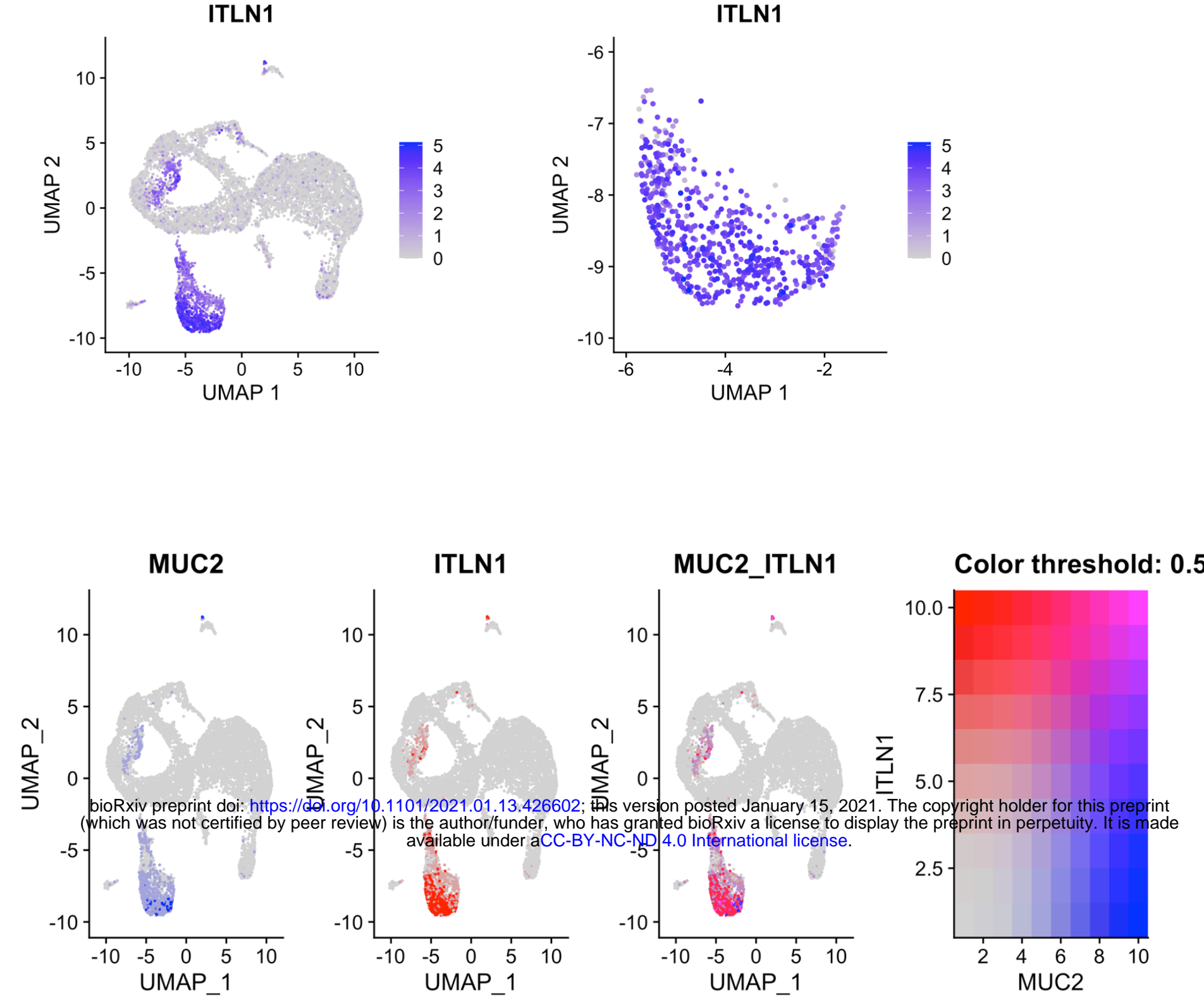


\section{Supplemental Table 1}

\begin{tabular}{|l|l|l|l|}
\hline Sample ID & Condition & Age & Sex \\
\hline 206 & NIBD & 55 & M \\
\hline 214 & NIBD & 64 & M \\
\hline 216 & NIBD & 63 & M \\
\hline 217 & NIBD & 72 & F \\
\hline 189 & CD & 25 & M \\
\hline 299 & CD & 56 & F \\
\hline 364 & CD & 68 & M \\
\hline
\end{tabular}

bioRxiv preprint doi: https://doi.org/10.1101/2021.01.13.426602; this version posted January 15, 2021. The copyright holder for this preprint (which was not certified by peer review) is the author/funder, who has granted bioRxiv a license to display the preprint in perpetuity. It is made available under aCC-BY-NC-ND 4.0 International license. 


\section{Supplemental Table 2}

\begin{tabular}{|c|c|c|c|c|c|c|}
\hline gene & p_val & avg_logFC & p_val_adj & exp_NIBD & exp_CD & $\log 2 \mathrm{FC}$ \\
\hline SLC26A2 & $4.35 \mathrm{E}-20$ & -0.40 & 0.00 & 16.95 & 11.06 & -0.62 \\
\hline CA2 & $4.77 \mathrm{E}-25$ & -0.39 & 0.00 & 23.00 & 15.18 & -0.60 \\
\hline ADIRF & $9.37 \mathrm{E}-09$ & -0.30 & 0.00 & 3.73 & 2.51 & -0.58 \\
\hline HSPA1B & $5.62 \mathrm{E}-12$ & -0.25 & 0.00 & 1.23 & 0.74 & -0.73 \\
\hline HIGD1A & $6.10 \mathrm{E}-07$ & -0.25 & 0.02 & 3.18 & 2.27 & -0.49 \\
\hline SLC20A1 & $6.22 \mathrm{E}-08$ & -0.22 & 0.00 & 3.69 & 2.77 & -0.41 \\
\hline GPA33 & $1.54 \mathrm{E}-07$ & -0.21 & 0.00 & 2.03 & 1.45 & -0.48 \\
\hline $\mathrm{SCIN}$ & 4.50E-09 & -0.21 & 0.00 & 0.83 & 0.48 & -0.78 \\
\hline SLC4A4 & $3.63 \mathrm{E}-08$ & -0.19 & 0.00 & 1.85 & 1.36 & -0.44 \\
\hline C3orf85 & $1.40 \mathrm{E}-07$ & -0.17 & 0.00 & 0.54 & 0.30 & -0.85 \\
\hline AC015912.3 & $6.87 \mathrm{E}-07$ & -0.17 & 0.02 & 0.40 & 0.18 & -1.13 \\
\hline NDUFC2 & $8.51 \mathrm{E}-07$ & -0.15 & 0.02 & 2.73 & 2.22 & -0.30 \\
\hline FASTKD1 & $6.05 \mathrm{E}-07$ & -0.11 & 0.01 & 0.27 & 0.13 & -1.01 \\
\hline MEF2C & $1.23 \mathrm{E}-08$ & -0.11 & 0.00 & 0.17 & 0.05 & -1.67 \\
\hline SLC35E2A & $1.32 \mathrm{E}-06$ & -0.05 & 0.03 & 0.06 & 0.01 & -3.16 \\
\hline GATD3A & $6.54 \mathrm{E}-07$ & 0.07 & 0.02 & 0.04 & 0.11 & 1.50 \\
\hline NR1/2 & $1.20 \mathrm{E}-06$ & 0.10 & 0.03 & 0.11 & 0.22 & 1.03 \\
\hline ZNRD2 & $1.17 \mathrm{E}-06$ & 0.10 & 0.03 & 0.16 & 0.28 & 0.85 \\
\hline TMEM120B & $6.62 \mathrm{E}-09$ & 0.10 & 0.00 & 0.08 & 0.20 & 1.30 \\
\hline TMC6 & $9.28 \mathrm{E}-07$ & 0.11 & 0.02 & 0.15 & 0.28 & 0.96 \\
\hline MAP1LC3A & $1.38 \mathrm{E}-06$ & 0.12 & 0.03 & 0.35 & 0.53 & 0.58 \\
\hline IMP4 & 5.99E-07 & 0.12 & 0.01 & 0.27 & 0.43 & 0.70 \\
\hline SNRK & 7.57E-07 & 0.12 & 0.02 & 0.27 & 0.44 & 0.68 \\
\hline SLC25A25 & $5.18 \mathrm{E}-07$ & 0.13 & 0.01 & 0.25 & 0.42 & 0.74 \\
\hline FUOM & $1.36 \mathrm{E}-06$ & 0.13 & 0.03 & 0.33 & 0.52 & 0.63 \\
\hline
\end{tabular}

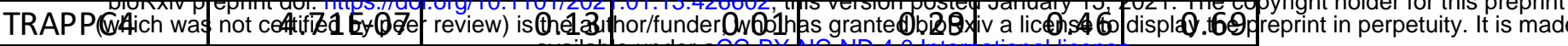
\begin{tabular}{|l|r|rr|r|r|r|}
\hline FERMT1 & $2.75 \mathrm{E}-07$ & 0.13 & 0.01 & 0.56 & 0.78 & 0.48 \\
\hline
\end{tabular}

\begin{tabular}{|c|c|c|c|c|c|c|}
\hline & & & & & & \\
\hline PSMD1 & $1.85 \mathrm{E}-07$ & 0.14 & 0.00 & 0.70 & 0.95 & 0.44 \\
\hline RHOF & $2.29 \mathrm{E}-07$ & 0.14 & 0.01 & 0.34 & 0.54 & 0.67 \\
\hline REL & 8.29E-09 & 0.14 & 0.00 & 0.25 & 0.44 & 0.83 \\
\hline RNASET2 & $7.75 \mathrm{E}-07$ & 0.15 & 0.02 & 0.72 & 1.00 & 0.48 \\
\hline GALNT5 & 7.33E-09 & 0.16 & 0.00 & 0.18 & 0.38 & 1.09 \\
\hline SLC7A1 & $3.48 \mathrm{E}-10$ & 0.16 & 0.00 & 0.28 & 0.50 & 0.86 \\
\hline KLF13 & $1.54 \mathrm{E}-07$ & 0.16 & 0.00 & 0.87 & 1.20 & 0.47 \\
\hline PRDM1 & $3.08 \mathrm{E}-07$ & 0.17 & 0.01 & 0.71 & 1.03 & 0.54 \\
\hline TOR1AIP2 & $4.03 \mathrm{E}-07$ & 0.17 & 0.01 & 0.69 & 1.01 & 0.54 \\
\hline RAB11FIP1 & $1.18 \mathrm{E}-07$ & 0.18 & 0.00 & 3.98 & 4.94 & 0.31 \\
\hline INPP1 & $1.83 \mathrm{E}-07$ & 0.18 & 0.00 & 0.52 & 0.82 & 0.66 \\
\hline QPRT & $2.34 \mathrm{E}-09$ & 0.19 & 0.00 & 0.17 & 0.41 & 1.29 \\
\hline EMP2 & 7.93E-07 & 0.19 & 0.02 & 1.14 & 1.59 & 0.47 \\
\hline NEDD4L & $1.30 \mathrm{E}-07$ & 0.19 & 0.00 & 0.96 & 1.38 & 0.53 \\
\hline ODC1 & $1.06 \mathrm{E}-06$ & 0.21 & 0.03 & 2.10 & 2.82 & 0.43 \\
\hline FRMD1 & 6.90E-09 & 0.23 & 0.00 & 0.22 & 0.53 & 1.28 \\
\hline CCND2 & $6.13 \mathrm{E}-09$ & 0.24 & 0.00 & 0.74 & 1.21 & 0.72 \\
\hline EFNA1 & $2.28 \mathrm{E}-11$ & 0.28 & 0.00 & 0.74 & 1.29 & 0.81 \\
\hline
\end{tabular}




\section{Supplemental Table 3}

\begin{tabular}{|c|c|c|c|c|c|c|}
\hline gene & p_val & avg_logFC & p_val_adj & exp_NIBD & exp_CD & $\log 2 \mathrm{FC}$ \\
\hline LGALS1 & $2.85 \mathrm{E}-07$ & -0.78 & 0.01 & 1.42 & 0.11 & -3.70 \\
\hline AQP8 & $1.58 \mathrm{E}-19$ & -0.73 & 0.00 & 60.74 & 28.71 & -1.08 \\
\hline IL32 & $1.60 \mathrm{E}-08$ & -0.51 & 0.00 & 19.62 & 11.35 & -0.79 \\
\hline AK1 & 7.45E-09 & -0.49 & 0.00 & 4.51 & 2.37 & -0.93 \\
\hline CFDP1 & $1.19 \mathrm{E}-10$ & -0.46 & 0.00 & 4.61 & 2.54 & -0.86 \\
\hline PPP1R14A & 2.09E-07 & -0.45 & 0.01 & 0.83 & 0.17 & -2.33 \\
\hline NQO1 & $1.42 \mathrm{E}-09$ & -0.45 & 0.00 & 1.85 & 0.81 & -1.18 \\
\hline RHOC & $1.02 \mathrm{E}-09$ & -0.43 & 0.00 & 8.50 & 5.20 & -0.71 \\
\hline CA4 & $1.68 \mathrm{E}-09$ & -0.41 & 0.00 & 22.72 & 14.66 & -0.63 \\
\hline DIO3OS & $8.15 \mathrm{E}-08$ & -0.38 & 0.00 & 0.61 & 0.09 & -2.68 \\
\hline CDKN2B-AS1 & $7.06 \mathrm{E}-08$ & -0.38 & 0.00 & 2.52 & 1.41 & -0.84 \\
\hline OAZ1 & $2.00 \mathrm{E}-08$ & -0.37 & 0.00 & 8.83 & 5.79 & -0.61 \\
\hline S100A13 & $1.61 \mathrm{E}-07$ & -0.35 & 0.00 & 0.86 & 0.31 & -1.46 \\
\hline FTH1 & $5.50 \mathrm{E}-08$ & -0.35 & 0.00 & 180.01 & 126.94 & -0.50 \\
\hline HSPB1 & 4.66E-07 & -0.35 & 0.01 & 4.69 & 3.02 & -0.63 \\
\hline SCNN1B & $1.56 \mathrm{E}-06$ & -0.35 & 0.04 & 1.16 & 0.52 & -1.14 \\
\hline NBL1 & $2.05 \mathrm{E}-07$ & -0.33 & 0.01 & 3.70 & 2.39 & -0.63 \\
\hline SPINT1-AS1 & $1.30 \mathrm{E}-06$ & -0.30 & 0.03 & 1.01 & 0.49 & -1.03 \\
\hline JUND & $7.32 \mathrm{E}-09$ & -0.29 & 0.00 & 14.86 & 10.92 & -0.44 \\
\hline GUCA2A & $6.48 \mathrm{E}-08$ & -0.28 & 0.00 & 51.86 & 38.99 & -0.41 \\
\hline CELA3B & $1.43 \mathrm{E}-06$ & -0.23 & 0.04 & 0.40 & 0.11 & -1.88 \\
\hline GUCA2B & $2.26 \mathrm{E}-10$ & -0.16 & 0.00 & 23.29 & 19.63 & -0.25 \\
\hline REG1A & $3.61 \mathrm{E}-09$ & 0.16 & 0.00 & 1.00 & 1.34 & 0.43 \\
\hline MT-ND2 & 8.83E-07 & 0.17 & 0.02 & 64.15 & 76.32 & 0.25 \\
\hline RPS6 $\begin{array}{c}\text { bioRxiv of } \\
\text { (which wa }\end{array}$ & 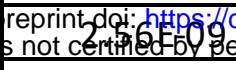 & $\begin{array}{l}\text { oi.org/10.11 } \\
\text { er review) is } 9 \mathrm{he}, 2\end{array}$ & $\begin{array}{l}1.01 .13 .426692 ; \\
\text { thor/funder; Who }\end{array}$ & $\begin{array}{l}\text { this version } \text { ses } \\
\text { has granted bto }\end{array}$ & \begin{tabular}{|l|} 
ed Januarys \\
Rxiv a license
\end{tabular} & \begin{tabular}{|l}
2021 \\
display \\
\end{tabular} \\
\hline RPL23 & $3.30 \mathrm{E}-07$ & ovajige & pe under $\mathrm{z} . \mathrm{G}-\mathrm{P}$ & P-NC-ND $4.99^{19^{t}}$ & inationaj li.24 & e. $\quad 0.76$ \\
\hline RPS7 & $3.95 \mathrm{E}-07$ & 0.39 & 0.01 & 3.55 & 5.74 & 0.69 \\
\hline RPL10A & 2.19E-09 & 0.51 & 0.00 & 2.87 & 5.41 & 0.92 \\
\hline FDPS & 8.94E-09 & 0.52 & 0.00 & 1.80 & 3.72 & 1.05 \\
\hline
\end{tabular}




\section{Supplemental Table 4}

\begin{tabular}{|r|r|r|r|c|}
\hline Sample ID & Condition & Avg. UMI per cell & Avg. genes per cell & $\begin{array}{c}\text { Avg. \% of mitochonodrial } \\
\text { reads per cell }\end{array}$ \\
\hline 206 & NIBD & 21670.1 & 4153.1 & 20.1 \\
\hline 214 & NIBD & 21562 & 4216.2 & 20.1 \\
\hline 216 & NIBD & 23015.5 & 4187.4 & 20.8 \\
\hline 217 & NIBD & 21538.3 & 4104.8 & 18.9 \\
\hline 189 & CD & 19781 & 3895.8 & 20.2 \\
\hline 299 & CD & 17240.9 & 3592.3 & 21.2 \\
\hline 364 & CD & 20114 & 3991.2 & 18.7 \\
\hline
\end{tabular}

bioRxiv preprint doi: https://doi.org/10.1101/2021.01.13.426602; this version posted January 15, 2021. The copyright holder for this preprint which was not certified by peer review) is the author/funder, who has granted bioRxiv a license to display the preprint in perpetuity. It is made
available under aCC-BY-NC-ND 4.0 International license. 